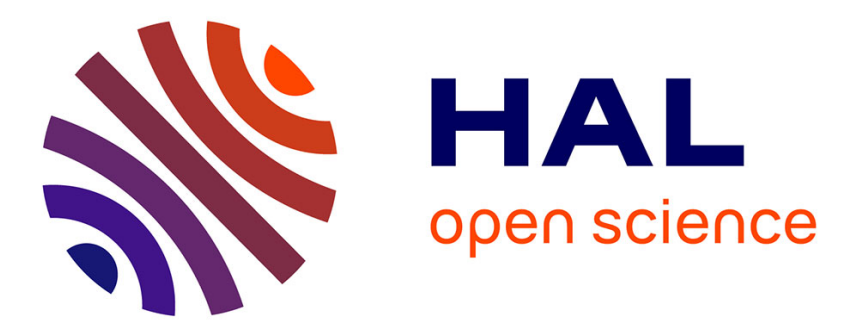

\title{
A model for elastic-viscoplastic deformations of crystalline solids based on material symmetry: Theory and plane-strain simulations
}

\author{
John Edmiston, David Steigmann, G. J. Johnson, N. Barton
}

\section{To cite this version:}

John Edmiston, David Steigmann, G. J. Johnson, N. Barton. A model for elastic-viscoplastic deformations of crystalline solids based on material symmetry: Theory and plane-strain simulations. International Journal of Engineering Science, 2013, 63, pp.10-22. hal-00782116

\author{
HAL Id: hal-00782116 \\ https://hal.science/hal-00782116
}

Submitted on 29 Jan 2013

HAL is a multi-disciplinary open access archive for the deposit and dissemination of scientific research documents, whether they are published or not. The documents may come from teaching and research institutions in France or abroad, or from public or private research centers.
L'archive ouverte pluridisciplinaire HAL, est destinée au dépôt et à la diffusion de documents scientifiques de niveau recherche, publiés ou non, émanant des établissements d'enseignement et de recherche français ou étrangers, des laboratoires publics ou privés. 
A model for elastic-viscoplastic deformations of crystalline solids based on material symmetry: Theory and plane-strain simulations

J. Edmiston ${ }^{1,2}$, D.J. Steigmann ${ }^{1 *}$, G.J. Johnson ${ }^{1}$ and N. Barton ${ }^{2}$

${ }^{1}$ Department of Mechanical Engineering

University of California

Berkeley, CA. 94720 USA

${ }^{2}$ Lawrence Livermore National Laboratory

Livermore, CA. 94550 USA

*author to receive correspondence (steigman@me.berkeley.edu) 
Abstract: A model for the elastic-viscoplastic response of metallic single crystals is developed on the basis of the modern finite-deformation theory of plasticity combined with considerations of material symmetry. This is proposed as an alternative to conventional crystal plasticity theory, based on a decomposition of the plastic deformation rate into a superposition of slips on active slip systems. A simple special case of the general theory, modeling evolving geometrically necessary dislocations and their effect on hardening, is developed and used as the basis of numerical experiments.

\section{Introduction}

In the conventional theory of crystal plasticity the rate of plastic deformation is assumed to be expressible as a superposition of simple shear rates. Thus,

$$
\dot{\mathbf{G}} \mathbf{G}^{-1}=\sum \nu_{i} \mathbf{s}_{i} \otimes \mathbf{n}_{i}
$$

in which $\mathbf{G}$ is the plastic part of the deformation gradient, $\nu_{i}$ are the slips and the $\mathbf{s}_{i}$ and $\mathbf{n}_{i}$ are orthonormal vectors specifying the $i$ th slip system. The sum ranges over the currently active slip systems. This decomposition, though widely adopted [1-4], has been criticized on the grounds that for finite deformations it cannot be associated with a sequence of simple shears unless these are restricted in a certain manner [5]. In particular, the order of the sequence generally affects the overall plastic deformation, a fact which is not reflected in (1). Because sequential slip cannot be ruled out a priori, we conclude that for finite deformations (1) fails to furnish a general representation of the plastic deformation due to slip. This situation is tacitly acknowledged in [2], where (1) is retained but referred to as the crystal hypothesis. Beyond this, it is known $[1,2]$ that the slips in (1) are not the material derivatives of physically meaningful quantities. This caveat does not apply in the case of small deformations, where the slips may be regarded as the material derivatives of the shears on the respective slip systems [2]. In [6] the theory of structured deformations [7] is used to derive conditions under which (1) yields an approximation to the deformation associated with a sequence of slips. Interestingly, it is found that such deformations are well approximated by (1) in face-centered cubic crystals [6,8], but the issue remains unresolved for the other crystal classes, including various subclasses of cubic symmetry. Criticism of (1) was offered by

Naghdi [9] in his review of the state of finite-strain plasticity theory, together with the suggestion that the vectors associated with slip systems should properly be regarded as directors in a formalism based on Cosserat theory. This suggestion was taken up in [10,11], using kinematic assumptions leading to a variant of (1), to obtain a formulation incorporating the effects of geometrically necessary dislocations. However, certain experimental observations reported in [12] raise concerns regarding the unqualified use of (1). We conclude that despite advances achieved on the basis of (1), the present state of the subject indicates that the latter does not enjoy the status of an unqualified kinematic representation of plastic deformation in crystalline solids, its widespread use notwithstanding. This is not to say that theories based on (1) are flawed from the viewpoint of quantitative or qualitative predictions, however. Indeed, we have used such a model [13] to benchmark the alternative model proposed in this work.

This state of affairs furnishes impetus for an alternative phenomenological framework based entirely 
on the continuum mechanics of crystalline media, as proposed independently in [14], [15] and [16], for example, and further developed in [17-19]. We pursue such a formulation in the present work, and use it to make a number of predictions based on numerical simulations. Our particular contribution to this framework is the detailed consideration of material symmetry and its role in the formulation of material response functions associated with the strain energy, the yield function, the flow rule and the plastic spin. For this purpose we confine attention in this work to cubic crystal symmetry. The particular model we develop has been benchmarked, for homogeneous, inertia-less deformations accompanying uniaxial tension, against a conventional model for cubic crystals based on (1) [13], which in turn has been calibrated against experimental data. However, we emphasize that our framework, based fundamentally on considerations of material symmetry, is applicable to crystal classes for which (1) has not rigorously been shown to be valid. Extensions to various crystal symmetries are discussed in [13]. Accordingly, the present approach offers the potential for extending crystal plasticity beyond the reach of the conventional theory based on (1), insofar as the latter has been rigorously established for finite deformations only in the case of face-centered cubic crystals [6]. Nevertheless we emphasize that our work is tentative in the sense that the experimental data needed to support or disprove our model are not currently available. Our motivation derives in part from the need for a straightforward and firmly grounded theoretical framework that can form the foundation for precisely such an experimental program.

We emphasize that the concept of slip is entirely extraneous to models of the kind considered here. Comparisons between the present theory and existing theories based on (1) should thus be confined to predictions that are accessible using both model types, such as an overall stress-strain diagram. It is in this sense that the present model has been benchmarked against a model based on (1). In particular, we are not proposing to replace models based on (1), but instead to offer an alternative framework in which questions surrounding (1) or similar decompositions do not arise. However, like the classical theories, in the present framework it is possible to model the evolution of lattice vectors. The predictions of the present model may therefore be assessed against X-ray diffraction data. This issue is discussed in forthcoming work.

We use standard notation such as $\mathbf{A}^{t}, \mathbf{A}^{-1}, \mathbf{A}^{*}, \operatorname{tr} \mathbf{A}$ and $J_{A}$. These are respectively the transpose, the inverse, the cofactor, the trace and the determinant of a tensor $\mathbf{A}$, regarded as a linear transformation from a three-dimensional vector space to itself, the latter being identified with the translation space of the usual three-dimensional Euclidean point space. We also use Orth to denote the group of orthogonal tensors, and Sym and $S k w$ the linear spaces of symmetric and skew tensors. The tensor product of 3 -vectors is indicated by interposing the symbol $\otimes$, and the Euclidean inner product of tensors $\mathbf{A}, \mathbf{B}$ is denoted by $\mathbf{A} \cdot \mathbf{B}=\operatorname{tr}\left(\mathbf{A} \mathbf{B}^{t}\right)$; the associated norm is $|\mathbf{A}|=\sqrt{\mathbf{A} \cdot \mathbf{A}}$. In terms of orthogonal components, $\mathbf{A} \cdot \mathbf{B}=A_{i j} B_{i j}$, wherein the usual summation rule is implied. For a fourth-order tensor $\mathcal{A}$, the notation $\mathcal{A}[\mathbf{B}]$ stands for the second-order tensor with orthogonal components $\mathcal{A}_{i j k l} B_{k l}$. The transpose $\mathcal{A}^{t}$ is defined by $\mathbf{B} \cdot \mathcal{A}^{t}[\mathbf{A}]=\mathbf{A} \cdot \mathcal{A}[\mathbf{B}]$, and $\mathcal{A}$ is said to possess major symmetry if $\mathcal{A}^{t}=\mathcal{A}$. If $\mathbf{A} \cdot \mathcal{A}[\mathbf{B}]=\mathbf{A}{ }^{t} \cdot \mathcal{A}[\mathbf{B}]$ and $\mathbf{A} \cdot \mathcal{A}[\mathbf{B}]=\mathbf{A} \cdot \mathcal{A}\left[\mathbf{B}^{t}\right]$ then $\mathcal{A}$ is said to possess minor symmetry. The notation $F_{\mathbf{A}}$ stands for the tensor-valued derivative of a scalar-valued function $F(\mathbf{A})$. 


\section{Summary of the basic theory}

In the purely mechanical theory considered, the relevant variables are the motion $\chi(\mathbf{x}, t)$ and the plastic deformation tensor $\mathbf{K}(\mathbf{x}, t)$, where $\mathbf{x}$ is the position of a material point in a fixed reference placement $\kappa_{r}$ of the body. The values $\mathbf{y}=\chi(\mathbf{x}, t)$ are the positions of these points at time $t$ and generate the current placement $\kappa_{t}$ of the body as $\mathbf{x}$ ranges over $\kappa_{r}$. The deformation gradient, $\mathbf{F}=\nabla \chi$, is assumed to be invertible with $J_{F}>0$. These variables are used to define the elastic deformation

$$
\mathbf{H}=\mathbf{F K} \text {. }
$$

We impose $J_{H}>0$ and conclude that $J_{K}>0$. The plastic deformation is the inverse of the more commonly used measure $\mathbf{G}$ :

$$
\mathbf{G}=\mathbf{K}^{-1} \text {. }
$$

The decomposition (2) is associated with a vector space $\kappa_{i}$ called the local intermediate configuration, which is mapped to the translation spaces of $\kappa_{r}$ and $\kappa_{t}$ by $\mathbf{K}$ and $\mathbf{H}$, respectively.

The elastic strain energy of the body is

$$
U=\int_{\kappa_{t}} \psi(\mathbf{H}) d v
$$

where $\psi$ is the spatial strain-energy density. Attention is confined to materially uniform bodies, exemplified by single crystals. These have the property that the strain-energy density does not depend explicitly on $\mathbf{x}$. However, most of the following discussion, concerned with local aspects of the theory, remains valid if this restriction is relaxed. We are concerned mainly with the constitutive structure of the theory and therefore restrict attention to smooth processes.

The local equations of motion, assuming negligible body force, are

$$
\operatorname{Div} \mathbf{P}=\rho_{r} \ddot{\mathbf{y}}, \quad \mathbf{P F}^{t} \in \text { Sym in } \kappa_{r},
$$

where $\mathbf{P}=\mathbf{T F}^{*}$ is the Piola stress, $\mathbf{T}$ is the Cauchy stress, $\rho_{r}$ is the mass density, Div is the spatial divergence (i.e., the divergence with respect to $\mathbf{x}$ ), superposed dots are used to denote material derivatives $(\partial / \partial t$ at fixed $\mathbf{x})$, and $\mathbf{b}$ is the body force per unit mass.

The strain-energy per unit volume of $\kappa_{i}$ is

$$
W(\mathbf{H})=J_{H} \psi(\mathbf{H}),
$$

and generates the Cauchy stress via the formula [17]

$$
\mathbf{T H}^{*}=W_{\mathbf{H}}
$$

Necessary and sufficient for the symmetry of $\mathbf{T}$ (cf. $(5)_{2}$ ) is that $W$ depend on $\mathbf{H}$ through the elastic Cauchy-Green deformation tensor [17]

$$
\mathbf{C}=\mathbf{H}^{t} \mathbf{H}
$$

Thus,

$$
W(\mathbf{H})=\hat{W}(\mathbf{C}) .
$$


Equation (7) then generates

$$
J_{H} \mathbf{T}=\mathbf{H S H}^{t}
$$

where $\mathbf{S}$ is the elastic 2nd Piola-Kirchhoff stress given by $\mathbf{S}=\hat{\mathbf{S}}(\mathbf{C})$, with

$$
\hat{\mathbf{S}}(\mathbf{C})=2 \hat{W}_{\mathbf{C}}
$$

If $\kappa_{i}$ is natural, in the sense that $\hat{\mathbf{S}}(\mathbf{I})=\mathbf{0}$, then realistic constitutive hypotheses for metallic crystals [17] associate it with an undistorted state of the underlying crystal lattice. Further, following standard practice in anisotropic elasticity theory [20], we regard the strain energy as an absolute scalar. The strain-energy function $\hat{W}$ is then subject to the restriction

$$
\hat{W}(\mathbf{C})=\hat{W}\left(\mathbf{R}^{t} \mathbf{C R}\right)
$$

where $\mathbf{R} \in$ Orth is an element of the symmetry group for the material (see [17-19] for further discussion). Using (11), it is straightforward to demonstrate that

$$
\hat{\mathbf{S}}\left(\mathbf{R}^{t} \mathbf{C R}\right)=\mathbf{R}^{t} \hat{\mathbf{S}}(\mathbf{C}) \mathbf{R}
$$

To make use of restrictions arising from material symmetry in crystalline solids, it is necessary to specify information about the undistorted lattice (Section 3). It is shown in [17] that undistorted $\kappa_{i}$ may be attained by an equilibrium (i.e., inertia-less) deformation of an arbitrarily small unloaded sub-body, granted the degree of smoothness required by the mean-stress theorem.

The sum of the kinetic and strain energies of an arbitrary part $p \subset \kappa_{t}$ of the body is

$$
\int_{\pi} \Phi d V ; \quad \Phi=\Psi+\frac{1}{2} \rho_{r}|\dot{\mathbf{y}}|^{2},
$$

where $\pi$, with piecewise smooth boundary $\partial \pi$, is the region occupied by $p$ in $\kappa_{r}$, and

$$
\Psi(\mathbf{F}, \mathbf{K})=J_{K}^{-1} W(\mathbf{F K})
$$

is the referential strain-energy density.

The dissipation, $\mathcal{D}$, is the difference between the mechanical power $P$ supplied to $p$ and the rate of change of the total energy in $p$. Thus,

$$
\mathcal{D}=P-\frac{d}{d t} \int_{\pi} \Phi d V .
$$

For smooth fields this is expressible in the form [18]

$$
\mathcal{D}=\int_{\pi} D d V
$$

where

$$
D=\mathcal{E} \cdot \dot{\mathbf{K}} \mathbf{K}^{-1}
$$

in which

$$
\mathcal{E}=\Psi \mathbf{I}-\mathbf{F}^{t} \mathbf{P}
$$

is Eshelby's energy-momentum tensor. The dissipation is thus non-negative for every sub-body if and only if $D \geq 0$. We assume plasticity to be strictly dissipative in the sense that $\dot{\mathbf{K}} \neq \mathbf{0}$ if and only if $D>0$. 
We find it convenient to use (18) in the form

$$
J_{K} D=\mathcal{E}^{\prime} \cdot \mathbf{K}^{-1} \dot{\mathbf{K}}
$$

where

$$
\mathcal{E}^{\prime}=J_{K} \mathbf{K}^{t} \mathcal{E} \mathbf{K}^{-t}
$$

is the Eshelby tensor, pushed forward to $\kappa_{i}$. This is purely elastic in origin; in particular [17],

$$
\mathcal{E}^{\prime}(\mathbf{C})=\hat{W}(\mathbf{C}) \mathbf{I}-\mathbf{C} \hat{\mathbf{S}}(\mathbf{C}),
$$

implying that

$$
\mathcal{E}^{\prime}\left(\mathbf{R}^{t} \mathbf{C R}\right)=\mathbf{R}^{t} \mathcal{E}^{\prime}(\mathbf{C}) \mathbf{R}
$$

if $\mathbf{R} \in$ Orth is a material symmetry transformation.

The equations of motion are augmented by a flow rule for the plastic deformation. Typically [17] this specifies $\mathbf{K}^{-1} \dot{\mathbf{K}}$ in terms of a constitutive response function, which must be such as to satisfy the material-symmetry transformation rule $\mathbf{K}^{-1} \dot{\mathbf{K}} \rightarrow \mathbf{R}^{t}\left(\mathbf{K}^{-1} \dot{\mathbf{K}}\right) \mathbf{R}$. A framework for rate-independent response is described in Section 4.

\section{Lattices}

In crystal-elasticity theory the stress arises in response to lattice distortion. This theory is based on the idea that linearly independent, undistorted lattice vectors $\mathbf{l}_{i}(i \in\{1,2,3\})$ are mapped to their images $\mathbf{t}_{i}$ in $\kappa_{t}$ in accordance with the Cauchy-Born hypothesis; that is, the $\mathbf{l}_{i}$ are convected as material vectors. To accommodate plasticity, this hypothesis is assumed to apply to the elastic deformation. Thus, $\mathbf{t}_{i}=\mathbf{H l}_{i}$ where $\mathbf{l}_{j}$ are the lattice vectors in $\kappa_{i}$. The lattice set $\left\{\mathbf{l}_{i}\right\}$ associated with $\kappa_{i}$ is assumed to be an intrinsic property of the material. Accordingly, it is regarded as a uniform field (i.e., independent of $\mathbf{x}$ ) in a materially-uniform body.

The $\mathbf{t}_{i}$ are observable in principle. In practice they are computed from their measurable duals $\mathbf{t}^{i}$ [21]. Eq. (2) yields $\mathbf{t}_{i}=\mathbf{F r}_{i}$, where $\mathbf{r}_{i}=\mathbf{K l}_{i}$ are the lattice vectors in $\kappa_{r}$. The plastic deformation is then given by $\mathbf{K}=\mathbf{r}_{i} \otimes \mathbf{l}^{i}$, where the $\mathbf{l}^{j}$ are the duals of the $\mathbf{l}_{j}$. The elastic deformation is given by $\mathbf{H}=\mathbf{t}_{i} \otimes \mathbf{l}^{i} ;$ and the deformation gradient by $\mathbf{F}=\mathbf{t}_{i} \otimes \mathbf{r}^{i}$.

The material derivatives of the referential lattice vectors are $\dot{\mathbf{r}}_{i}=\dot{\mathbf{K}} \mathbf{l}_{i}+\mathbf{K} \mathbf{i}_{i}$. These imply that if $\mathbf{i}_{i} \neq \mathbf{0}$, then the lattice vectors are non-material $\left(\dot{\mathbf{r}}_{i} \neq \mathbf{0}\right)$ in the absence of plastic flow $(\dot{\mathbf{K}}=\mathbf{0})$, contrary to widespread opinion to the effect that plastic flow is solely responsible for the non-materiality of the lattice; i.e., that plastic flow alone accounts for the evolution of material vectors relative to the lattice. Thus we impose $\dot{\mathbf{l}}_{i}=\mathbf{0}$ and regard the set $\left\{\mathbf{l}_{i}\right\}$ of lattice vectors as assigned data. This in turn yields the materiality of the set $\left\{\mathbf{r}_{i}\right\}$ in the absence of plastic flow, in accordance with the conventional statement of the Cauchy-Born hypothesis for elastic deformations.

This issue bears directly on the notion of plastic spin. In general this cannot be suppressed if the orientation of the intermediate lattice is fixed [19], as assumed here. Particular attention is thus devoted to plastic spin in the present work. 
There is a degree of arbitrariness in this formulation associated with the (fixed) orientation of the undistorted lattice $\left\{\mathbf{l}_{i}\right\}$. In [18] it is shown that the local differential equations associated with theories of the kind considered here are insensitive to this orientation. However, the latter manifests itself via boundary conditions on position and traction, and is therefore not entirely arbitrary. A similar observation applies to the conventional theory of crystal plasticity in which the slip-system vectors are specified as data. In practice this means that $\left\{\mathbf{l}_{i}\right\}$ is also specified. If data are available for the lattice $\left\{\mathbf{r}_{i}\right\}$ at some initial instant, as the present theory requires, then the initial value of plastic deformation is thereby determined. Often, the initial instant is presumed to correspond to a virgin state, at which the plastic deformation reduces to the identity. In this case the undistorted lattice is measurable in principle, as it then coincides with $\left\{\mathbf{r}_{i}\right\}$. Fixing $\left\{\mathbf{l}_{i}\right\}$ is thus tantamount to the assertion that a virgin state can be identified, and hence that the (uniform) undistorted lattice is experimentally accessible.

\section{Rate-independent theory and a natural viscoplastic extension}

We consider the rate independent theory in detail. Our purpose is to outline the basic structure of the theory and to use it as the basis of a natural rate-dependent extension, in the manner of the conventional extensions of classical rate-independent theory [22] to model viscoplastic behavior.

Following conventional ideas for the description of rate-independent response we assume plastic flow to be possible only if the material is in a state of yield. Specifically we require that the elastic deformation belong to a manifold that may be parametrized by other variables. For example, motivated by G.I. Taylor's formula giving the flow stress as a function of dislocation density, and using the fact that the stress $\mathbf{S}$ may be expressed in terms of $\mathbf{C}$ via (11), we assume yield to be possible only if $[17,18]$

$$
G(\mathbf{C}, \alpha)=0
$$

where $G$ is a suitable yield function and

$$
\alpha=J_{K} \mathbf{K}^{-1} \operatorname{Curl} \mathbf{K}^{-1}
$$

is the (geometrically necessary) dislocation density. Here Curl is the referential curl operation defined in terms of the usual vector operation by

$$
(\operatorname{Curl} \mathbf{A}) \mathbf{c}=\operatorname{Curl}\left(\mathbf{A}^{t} \mathbf{c}\right)
$$

for any fixed vector c. Relevant to our development is the current yield surface, defined, for fixed $\alpha$, by $G(\cdot, \alpha)=0$. For simplicity's sake we assume $G$ to be differentiable, so that the yield surface defines a differentiable manifold in Sym.

As is well known the identification of dislocations as either geometrically necessary and statistically stored in any theory of plastic flow is an inherently scale-dependent matter which reflects the length scales at which the model purports to be descriptive. Here we make the role of geometrically necessary dislocations explicit, whereas the role of statistically stored dislocations is relegated to the details of the structure of the flow rule itself, in the form of the particular function used as well as the scale-dependent material properties occurring within it. It is our view that the assessment of the length scales at which 
a particular model is descriptive, if any, cannot be decided a priori and hence that recourse must be made to experiments.

Plastic evolution; i.e., $\dot{\mathbf{K}} \neq \mathbf{0}$, is deemed to be possible only when $(24)$ is satisfied, and the variable $\mathbf{C}$ is always constrained to belong to the current elastic range defined by $G(\cdot, \alpha) \leq 0$, assumed to be a connected set in Sym. In view of our restriction to materially uniform bodies we require that the same yield function pertain to all material points.

In [17] it is shown that (24) is invariant under superposed rigid-body motions and (global) changes of reference placement and is thus intrinsic to the material, provided that the function G is likewise invariant. Similar statements apply to the reduced strain-energy function (9) and to the associated stress, given by (11). In particular, the stated invariance properties are possessed by the tensors $\mathbf{C}$ and $\alpha[10,17]$. Further, the yield function is subject to the same material-symmetry restriction as that imposed on the strain-energy function; i.e. [17],

$$
G(\mathbf{C}, \alpha)=G\left(\mathbf{R}^{t} \mathbf{C R},(\operatorname{det} \mathbf{R}) \mathbf{R}^{t} \alpha \mathbf{R}\right)
$$

obtained by regarding the values of $G$ as absolute scalars. It is important to note that the dislocation density is well-defined under symmetry transformations only if the symmetry group is discrete (see Theorem 8 of [24]). Accordingly, yield functions of the kind considered are meaningful only for crystalline solids.

The body is dislocated if $\alpha$ does not vanish; in this case $\mathbf{K}^{-1}$ is not a gradient and from (2) it follows that neither is $\mathbf{H}$. In fact [23],

$$
\alpha=J_{H} \mathbf{H}^{-1} \operatorname{curl} \mathbf{H}^{-1}
$$

in which curl is the spatial curl. Then, $\kappa_{i}$ has only local significance in the sense that it cannot be identified with a global placement of the body in Euclidean space. That is, a differentiable position field that identifies material points in $\kappa_{i}$ does not exist.

In the setting of the rate-independent theory, most workers assume the plastic evolution $\mathbf{K}^{-1} \dot{\mathbf{K}}$ to be such as to maximize the dissipation under the constraint that $\mathbf{C}$ belong to the current yield surface [25]. This in turn is a provable consequence of the widely adopted I'llyushin postulate [26]. In the present context this condition takes the form [18]

$$
\left[\mathcal{E}^{\prime}(\mathbf{C})-\mathcal{E}^{\prime}\left(\mathbf{C}^{*}\right)\right] \cdot \mathbf{K}^{-1} \dot{\mathbf{K}} \geq 0 ; \quad G(\mathbf{C}, \alpha)=0
$$

where $\mathbf{C}^{*}$ is a fixed elastic deformation in the elastic range. This inequality is invariant under material symmetry transformations.

The requirement that the elastic distortion be confined to the current elastic range implies, for typical metallic crystals, that the associated strain

$$
\mathbf{E}=\frac{1}{2}(\mathbf{C}-\mathbf{I})
$$

is small enough to justify the use of the linear relation

$$
\mathbf{S}=\mathcal{C}[\mathbf{E}]
$$


where $\mathcal{C}$ is the (fixed) tensor of elastic moduli pertaining to the crystalline solid at hand. This possesses both major and minor symmetry and is normally taken to be positive definite on the linear space of symmetric tensors. Accordingly, the strain energy is approximated by

$$
\hat{W} \simeq \tilde{W}(\mathbf{E})=\frac{1}{2} \mathbf{E} \cdot \mathcal{C}[\mathbf{E}]
$$

whereas (22) and (30) yield $\mathcal{E}^{\prime}=-\mathbf{S}+o(|\mathbf{E}|)$. Then, (29) is approximated to leading order by

$$
\left[\mathbf{S}(\mathbf{E})-\mathbf{S}\left(\mathbf{E}^{*}\right)\right] \cdot \dot{\mathbf{G}} \mathbf{G}^{-1} \geq 0
$$

Our constitutive hypothesis also implies that $\mathbf{S}(\mathbf{E})$ is invertible, and hence that the yield function may be expressed in terms of $\mathbf{S}$ instead of $\mathbf{E}$. We write

$$
F(\mathbf{S}, \alpha)=G(\mathbf{E}(\mathbf{S}), \alpha)
$$

where $\mathbf{E}(\cdot)$ is the inverse of the function $\mathbf{S}(\cdot)$. The current yield function is

$$
H(\cdot)=F(\cdot, \alpha)
$$

and the current elastic range is defined by $H \leq 0$.

To the same order in elastic strain, the dissipation inequality $D \geq 0$ is reduced, with the aid of (20) and (22), to the statement

$$
\mathbf{S} \cdot \dot{\mathbf{G}} \mathbf{G}^{-1} \geq 0 \text { for all } \dot{\mathbf{G}}
$$

and the strong dissipation hypothesis $D>0$ associated with actual plastic evolution reduces to

$$
\mathbf{S} \cdot \dot{\mathbf{G}} \mathbf{G}^{-1}>0 \text { if and only if } \dot{\mathbf{G}} \neq \mathbf{0} \text {. }
$$

Thus the problem is to characterize the plastic flow such that the actual dissipation is maximized relative to that associated with any admissible state; i.e.,

$$
\max \left(\mathbf{S} \cdot \dot{\mathbf{G}} \mathbf{G}^{-1}\right) \quad \text { subject to } \quad F(\mathbf{S}, \alpha) \leq 0 \quad \text { and } \quad \mathbf{W}=\mathbf{0}, \quad \text { where } \quad \mathbf{W}=S k w \mathbf{S},
$$

which is a standard optimization problem subject to equality and inequality constraints. The KuhnTucker necessary condition [27] immediately generates the flow rule

$$
\dot{\mathbf{G}} \mathbf{G}^{-1}=(\lambda F+\overline{\mathbf{\Omega}} \cdot \mathbf{W})_{\mathbf{S}}
$$

where $\lambda \in \mathbb{R}^{+}$and $\overline{\boldsymbol{\Omega}} \in S k w$ are Lagrange multipliers. It is straightforward to derive $(\overline{\boldsymbol{\Omega}} \cdot \mathbf{W})_{\mathbf{S}}=\overline{\boldsymbol{\Omega}}$ and thereby obtain

$$
\dot{\mathbf{G}} \mathbf{G}^{-1}=\lambda F_{\mathbf{S}}+\bar{\Omega} .
$$

From (20) the dissipation is given, to leading order in the elastic strain, by

$$
J_{K} D=\lambda \mathbf{S} \cdot F_{\mathbf{S}}
$$

Because $\lambda \geq 0$, the dissipation is strictly positive only if $\lambda>0$ and hence only if

$$
\mathbf{S} \cdot F_{\mathbf{S}}>0
$$


Moreover, given $\overline{\boldsymbol{\Omega}}$ we may always find $\boldsymbol{\Omega} \in S k w$ such that $\overline{\boldsymbol{\Omega}}=\lambda \boldsymbol{\Omega}$ and recast (40) as

$$
\dot{\mathbf{G}} \mathbf{G}^{-1}=\lambda\left(F_{\mathbf{S}}+\mathbf{\Omega}\right) ; \quad F(\mathbf{S}, \alpha)=0 .
$$

We observe that material symmetry transformations yield $F_{\mathbf{S}} \rightarrow \mathbf{R}^{t} F_{\mathbf{S}} \mathbf{R}$; eq. (43) then requires that $\lambda$ be invariant and that $\boldsymbol{\Omega} \rightarrow \mathbf{R}^{t} \boldsymbol{\Omega} \mathbf{R}$. Further, when the yield function depends on the dislocation density the consistency condition associated with continued yielding generates a partial differential equation for the Lagrange multiplier $\lambda$ rather than the algebraic equation associated with conventional theories [18]. Indeed, the presence of spatial derivatives in the flow rule, due to the dependence of the yield function on the dislocation density, raises mathematical issues in the rate-independent theory that do not arise in conventional treatments of rate-independent response. These furnish impetus for further study of the present model. Similar issues have been identified, in the context of finite-element implementation, in $[10,11]$. Partly to avoid these in the present, preliminary, work, we confine our further attention to a rate-dependent viscoplastic extension of the theory. This is integrated, in Section 6, using explicit forward-differencing in time. In this scheme the initial and boundary conditions suffice to specify the evolving solution completely, whether or not spatial derivatives are involved in the yield function.

In the spirit of classical theories of viscoplasticity [22] we propose the simple extension

$$
\dot{\mathbf{G}} \mathbf{G}^{-1}=\nu^{-1}\left(F_{\mathbf{S}}+\boldsymbol{\Omega}\right) ; \quad F(\mathbf{S}, \alpha) \geq 0
$$

to model rate-dependence, where $\nu(>0)$ is a material viscosity coefficient. This is far simpler than the rate-independent theory, for two reasons: First, there is no need to ensure that the state of stress associated with a given dislocation density remains confined to the elastic range; and second, there is no consistency condition and hence no need to solve a partial differential equation for a multiplier field $\lambda$. This is replaced in the viscoplastic framework by an assigned viscosity.

\section{A model for cubic crystals}

In this section we develop constitutive equations for cubic crystals. The phenomenological nature of the theory and the paucity of detailed experimental data mean that there is enormous latitude in proposing specific forms. To render the problem tractable, we impose certain a prior conditions in addition to those required by material symmetry.

\subsection{Strain-energy and yield functions}

For cubic crystals the strain-energy function $W$ and the yield function $F$ are invariant under the group consisting of the rotations, reflections and inversions that map a cube to itself. To specify $W$ we require the invariant functions that are homogeneous of degree two in the elastic strain $\mathbf{E}$, these being common to each of the five subclasses of cubic symmetry. They are [28]

$$
(\operatorname{tr} \mathbf{E})^{2}, \quad E_{11} E_{22}+E_{11} E_{33}+E_{22} E_{33} \quad \text { and } \quad E_{12}^{2}+E_{13}^{2}+E_{23}^{2}
$$

where $E_{i j}=\mathbf{E} \cdot \operatorname{Sym}\left(\mathbf{e}_{i} \otimes \mathbf{e}_{j}\right)$ and $\left\{\mathbf{e}_{i}\right\}$ is an orthonormal basis aligned with the cube axes (i.e.; with the elements of the set $\left\{\mathbf{l}_{i}\right\}$ of lattice vectors, or their duals). We emphasize the fact that the orthonormal 
basis, and hence the orientation of the cube, must be specified. It is regarded as remaining fixed at the material point in question, and, for materially-uniform bodies, carried over to all such points.

Some simplification is achieved by using the deviatoric strain $\mathbf{E}$. This yields

$$
E_{11} E_{22}+E_{11} E_{33}+E_{22} E_{33}=\frac{1}{3}(\operatorname{tr} \mathbf{E})^{2}-\frac{1}{2}\left(\bar{E}_{11}^{2}+\bar{E}_{22}^{2}+\bar{E}_{33}^{2}\right)
$$

and so the strain-energy function is expressible in the form [18]

$$
\tilde{W}(\mathbf{E})=\frac{1}{2}\left[C_{1}(\operatorname{tr} \mathbf{E})^{2}+C_{2}\left(\bar{E}_{11}^{2}+\bar{E}_{22}^{2}+\bar{E}_{33}^{2}\right)\right]+C_{3}\left(E_{12}^{2}+E_{13}^{2}+E_{23}^{2}\right),
$$

where $C_{i}$ are the (constant) moduli, giving the strain energy as a linear combination of three independent quadratic forms. Because each is positive definite, the relevant constitutive hypothesis is met if and only if each $C_{i}>0$. The associated stress is [18]

$$
\begin{aligned}
\tilde{\mathbf{S}}(\mathbf{E})= & C_{1}(\operatorname{tr} \mathbf{E}) \mathbf{I}+C_{2}\left(\bar{E}_{11} \mathbf{e}_{1} \otimes \mathbf{e}_{1}+\bar{E}_{22} \mathbf{e}_{2} \otimes \mathbf{e}_{2}+\bar{E}_{33} \mathbf{e}_{3} \otimes \mathbf{e}_{3}\right) \\
& +C_{3}\left[E_{12}\left(\mathbf{e}_{1} \otimes \mathbf{e}_{2}+\mathbf{e}_{2} \otimes \mathbf{e}_{1}\right)+E_{13}\left(\mathbf{e}_{1} \otimes \mathbf{e}_{3}+\mathbf{e}_{3} \otimes \mathbf{e}_{1}\right)+E_{23}\left(\mathbf{e}_{2} \otimes \mathbf{e}_{3}+\mathbf{e}_{3} \otimes \mathbf{e}_{2}\right)\right] .
\end{aligned}
$$

Turning to the yield function, we simplify matters by assuming that its dependence on stress and dislocation density can be decoupled. Mainly for illustrative purposes, we also adopt the widespread assumption that pressure, proportional to $\operatorname{tr} \mathbf{T}$, has negligible effect on yield, this effectively limiting the applicability of the model to circumstances in which the pressure is not too large. To examine the implications, we use (8) and (10) to obtain $\operatorname{tr} \mathbf{T}=J_{H}^{-1} \operatorname{tr}(\mathbf{C S})$; eqs. (30) and (31) then furnish $\operatorname{tr} \mathbf{T}=\operatorname{tr} \mathbf{S}+o(|\mathbf{E}|)$. Consistency with our previous assumptions thus implies that the current yield function $H(\cdot)=F(\cdot, \alpha)$ should depend on $\mathbf{S}$ through its deviatoric part $\overline{\mathbf{S}}$; we write $H(\mathbf{S})=\bar{F}(\overline{\mathbf{S}})$. To represent this function we require the scalar invariants of $\overline{\mathbf{S}}$ under the cubic symmetry group through quadratic order (cf. (31)). The only linear invariant, $\operatorname{tr} \overline{\mathbf{S}}$, vanishes identically, leading to a function homogeneous of degree two:

$$
\bar{F}(\overline{\mathbf{S}})=\frac{1}{2} A_{1}\left(\bar{S}_{11}^{2}+\bar{S}_{22}^{2}+\bar{S}_{33}^{2}\right)+A_{2}\left(S_{12}^{2}+S_{13}^{2}+S_{23}^{2}\right),
$$

where $A_{1,2}$ are constants. Then,

$$
\begin{aligned}
F_{\mathbf{S}}= & A_{1}\left(\bar{S}_{11} \mathbf{e}_{1} \otimes \mathbf{e}_{1}+\bar{S}_{22} \mathbf{e}_{2} \otimes \mathbf{e}_{2}+\bar{S}_{33} \mathbf{e}_{3} \otimes \mathbf{e}_{3}\right) \\
& +A_{2}\left[S_{12}\left(\mathbf{e}_{1} \otimes \mathbf{e}_{2}+\mathbf{e}_{2} \otimes \mathbf{e}_{1}\right)+S_{13}\left(\mathbf{e}_{1} \otimes \mathbf{e}_{3}+\mathbf{e}_{3} \otimes \mathbf{e}_{1}\right)+S_{23}\left(\mathbf{e}_{2} \otimes \mathbf{e}_{3}+\mathbf{e}_{3} \otimes \mathbf{e}_{2}\right)\right],
\end{aligned}
$$

and we obtain

$$
\mathbf{S} \cdot F_{\mathbf{S}}=A_{1}|\overline{\mathbf{S}}|^{2}+2\left(A_{2}-A_{1}\right)\left(S_{12}^{2}+S_{13}^{2}+S_{23}^{2}\right) .
$$

Inequality (42) is then satisfied if and only if $\overline{\mathbf{S}} \neq \mathbf{0}$ and

$$
A_{1}>0, \quad A_{2}>A_{1} .
$$

We observe, from (44) and (50), that $t r \dot{\mathbf{G}} \mathbf{G}^{-1}=0$ and thus that $J_{G}$ is constant.

It follows from (52) that $\bar{F}(\cdot)$ is positive definite. To allow for yielding in a manner consistent with our assumptions, it is thus necessary that the yield function be expressible in the form

$$
F(\mathbf{S}, \alpha)=\bar{F}(\overline{\mathbf{S}})-K(\alpha),
$$


where $K(\cdot)$ is positively valued function subject to the material-symmetry restriction $K(\alpha)=K\left((\operatorname{det} \mathbf{R}) \mathbf{R}^{t} \alpha \mathbf{R}\right)$. The form that this function should take in applications to real crystals remains open. One possibility, compatible with cubic symmetry, is

$$
K=k_{0}\left(1+k_{1}|\alpha|^{2}\right)^{p},
$$

where $p, k_{0,1}$ are positive constants. This expression furnishes a purely phenomenological model of hardening of the crystal due to the presence of dislocations.

\subsection{Plastic spin}

The plastic spin plays the role of a Lagrange multiplier in the problem of maximizing dissipation in the rate-independent theory. It's role there is merely to determine the algebraic structure of the flow rule for plastic evolution. To obtain a complete theory it is necessary to augment this structure with a constitutive specification of plastic spin. The conventional theory of crystal viscoplasticity, based on (1) in conjunction with the flow rule [29],

$$
\nu_{i}=\nu_{0}\left(\left|\tau^{(i)}\right| / s^{(i)}\right)^{1 / m} \operatorname{sgn}\left(\tau^{(i)}\right) ; \quad\left|\tau^{(i)}\right|>s^{(i)},
$$

offers some guidance in this regard. Here $\tau^{(i)}$ is the resolved shear stress on the $i$ th slip system, $s^{(i)}$ is the associated yield value, and $\nu_{0}, m$ are material parameters. The main qualitative feature of this model is the prediction that plastic spin reverses upon reversal of the sign of the stress, which comports with the observed phenomenology [13]. Guided by this feature of the conventional theory, here we take the spin $\boldsymbol{\Omega}$ to depend only on $\mathbf{S}$ and to be such that

$$
\boldsymbol{\Omega}(-\mathbf{S})=-\mathbf{\Omega}(\mathbf{S})
$$

subject to the material-symmetry condition

$$
\boldsymbol{\Omega}\left(\mathbf{R}^{t} \mathbf{S R}\right)=\mathbf{R}^{t} \boldsymbol{\Omega}(\mathbf{S}) \mathbf{R}
$$

which reflects the influence of the structure of the underlying lattice. The influence of the lattice on plastic spin is of course explicit in the conventional theory based on (1) and (55).

Specific constitutive functions satisfying cubic symmetry are generated by assuming polynomial dependence on stress and generating an associated integrity basis using the methods outlined in $[28,30]$. Pursuant to this we note that there are no purely linear terms that satisfy (56) together with skew symmetry and so it necessary to retain terms through cubic order at least. Here we are interested in the simplest model that satisfies our requirements and thus terminate the polynomial at cubic order.

All tensor and vector components are referred to the normalized rectangular cubic lattice basis $\left\{\mathbf{e}_{i}\right\}$. We introduce a tensor $\mathbf{V}$ with components $V_{i j}$, form the scalar $\Omega_{i j} V_{i j}$ and define the matrices $\bar{\Omega}_{i j}=Q_{i k} \Omega_{k l} Q_{j l}$ and $\bar{V}_{i j}=Q_{i k} V_{k l} Q_{j l}$ with $\mathbf{Q}=\mathbf{R}^{t}$, where $\mathbf{R}$ belongs to the particular cubic symmetry group in question, assumed here to be the hexoctahedral group for the sake of definiteness. This is the subgroup of the cubic group associated with maximal symmetry, in the sense that it contains the maximum number of distinct elements. The latter are listed explicitly in Table 1 of [28]. Accordingly,

$$
\Omega_{i j}(\overline{\mathbf{S}}) \bar{V}_{i j}=Q_{i k} \Omega_{k l}(\mathbf{S}) Q_{j l} \bar{V}_{i j}=\Omega_{k l}(\mathbf{S}) V_{k l}
$$


where $\overline{\mathbf{S}}=\mathbf{Q S Q}^{t}$. The function

$$
Z\left(V_{i j}, \mathbf{S}\right)=\Omega_{i j}(\mathbf{S}) V_{i j}
$$

is thus invariant under the symmetry group. Further, because $\boldsymbol{\Omega}$ is skew, this may be written as

$$
Z\left(v_{i}, \mathbf{S}\right)=2 \omega_{i}(\mathbf{S}) v_{i}
$$

where $\omega$ and $\mathbf{v}$ are the axial vectors of $\boldsymbol{\Omega}$ and $S k w \mathbf{V}$, respectively, yielding [30]

$$
\omega_{k}=\frac{1}{2} \partial Z / \partial v_{k}
$$

In view of (60) we require only those integrity-basis elements that are linear in the axial vector $\mathbf{v}$. Among these we retain those through the third order in $\mathbf{S}$; namely,

$$
x=S_{11}+S_{22}+S_{33}, \quad y=S_{11}\left(S_{13} v_{2}-S_{12} v_{3}\right)+S_{22}\left(S_{12} v_{3}-S_{23} v_{1}\right)+S_{33}\left(S_{23} v_{1}-S_{13} v_{2}\right),
$$

and

$$
\begin{aligned}
z_{1} & =S_{22} S_{33}\left(S_{13} v_{2}-S_{12} v_{3}\right)+S_{33} S_{11}\left(S_{12} v_{3}-S_{23} v_{1}\right)+S_{11} S_{22}\left(S_{23} v_{1}-S_{13} v_{2}\right), \\
z_{2} & =S_{23}^{2}\left(S_{13} v_{2}-S_{12} v_{3}\right)+S_{13}^{2}\left(S_{12} v_{3}-S_{23} v_{1}\right)+S_{12}^{2}\left(S_{23} v_{1}-S_{13} v_{2}\right), \\
z_{3} & =S_{11}\left(S_{23} S_{12} v_{2}-S_{23} S_{13} v_{3}\right)+S_{22}\left(S_{23} S_{13} v_{3}-S_{12} S_{13} v_{1}\right)+S_{33}\left(S_{12} S_{13} v_{1}-S_{23} S_{12} v_{2}\right) .
\end{aligned}
$$

The derivation of this basis is quite involved and thus not presented here. A detailed derivation is provided in [13]. This accounts for the non-standard transformation rule $\mathbf{a} \rightarrow(\operatorname{det} \mathbf{Q}) \mathbf{Q a}$ obeyed by axial vectors. For our present purposes it suffices simply to note that these functions are invariant under the hexoctahedral group and thus meet the stated symmetry requirement. To ensure that $Z\left(v_{i},-\mathbf{S}\right)=$ $-Z\left(v_{i}, \mathbf{S}\right)$ in accordance with $(56)$, we have

$$
Z\left(v_{i}, \mathbf{S}\right)=B_{0} x y+\sum_{k=1}^{3} B_{i} z_{i}
$$

where $B_{0}$ and $B_{i}$ are material constants. The explicit form of $\boldsymbol{\Omega}$ is then given by (61) and

$$
\boldsymbol{\Omega}(\mathbf{S})=e_{j i k} \omega_{k} \mathbf{e}_{i} \otimes \mathbf{e}_{j}
$$

where $e_{j i k}$ is the usual permutation symbol $\left(e_{123}=+1\right)$, with

$$
\begin{aligned}
2 \omega_{1}= & B_{0}\left(S_{11}+S_{22}+S_{33}\right) S_{23}\left(S_{33}-S_{22}\right)+B_{1} S_{11} S_{23}\left(S_{22}-S_{33}\right) \\
& +B_{2} S_{23}\left(S_{12}^{2}-S_{13}^{2}\right)+B_{3} S_{12} S_{13}\left(S_{33}-S_{22}\right), \\
2 \omega_{2}= & B_{0}\left(S_{11}+S_{22}+S_{33}\right) S_{13}\left(S_{11}-S_{33}\right)+B_{1} S_{22} S_{13}\left(S_{33}-S_{11}\right) \\
& +B_{2} S_{13}\left(S_{23}^{2}-S_{12}^{2}\right)+B_{3} S_{12} S_{23}\left(S_{11}-S_{33}\right), \\
2 \omega_{3}= & B_{0}\left(S_{11}+S_{22}+S_{33}\right) S_{12}\left(S_{22}-S_{11}\right)+B_{1} S_{33} S_{12}\left(S_{11}-S_{22}\right) \\
& +B_{2} S_{12}\left(S_{13}^{2}-S_{23}^{2}\right)+B_{3} S_{13} S_{23}\left(S_{22}-S_{11}\right) .
\end{aligned}
$$

Estimates of the parameters $B_{0}-B_{3}$ are obtained in [13] by optimizing the present flow rule against the predictions of overall stress-strain diagrams using the standard crystal-plasticity model based on (1) and (55). 


\section{Plane-strain simulations}

We confine our further attention to the plane-strain theory for purposes of illustration. This is most easily expressed in Cartesian coordinates, using as basis the fixed orthonormal triad $\left\{\mathbf{e}_{\alpha}, \mathbf{k}\right\}$ aligned with the specified cubic lattice vectors $\left\{\mathbf{l}_{i}\right\}$, where $\mathbf{k}\left(=\mathbf{e}_{3}\right)$ is the unit normal to the considered plane. The non-zero elements of the plastic deformation $\mathbf{K}$ in this basis are $K_{\alpha \beta}\left(x_{1}, x_{2}\right)$ and $K_{33}=1$, where $x_{\alpha}$ are the in-plane Cartesian coordinates associated with the lattice vectors. Further, the non-zero components of $\mathbf{S}$ in the lattice basis are

$$
S_{11}=A E_{11}+B E_{22}, \quad S_{22}=B E_{11}+A E_{22}, \quad S_{12}=C E_{12} \quad \text { and } \quad S_{33}=B\left(E_{11}+E_{22}\right),
$$

where $E_{\alpha \beta}=\mathbf{E} \cdot \operatorname{Sym}\left(\mathbf{e}_{\alpha} \otimes \mathbf{e}_{\beta}\right)$ and

$$
A=C_{1}+\frac{2}{3} C_{2}, \quad B=C_{1}-\frac{1}{3} C_{2} \quad \text { and } \quad C=C_{3} .
$$

This implies that the axial plastic-spin vector has one non-zero component, given by

$$
2 \omega_{3}=B_{0}\left(S_{11}+S_{22}+S_{33}\right) S_{12}\left(S_{22}-S_{11}\right)+B_{1} S_{33} S_{12}\left(S_{11}-S_{22}\right)
$$

and hence that $\boldsymbol{\Omega}=\Omega_{\alpha \beta} \mathbf{e}_{\alpha} \otimes \mathbf{e}_{\beta}$ with $\Omega_{\alpha \beta}=\varepsilon_{\beta \alpha} \omega_{3}$, where $\varepsilon_{\beta \alpha}=e_{\beta \alpha 3}$. The appropriate specialization of the flow rule (44) is obtained by using (cf. (50))

$$
F_{\mathbf{S}}=A_{1}\left(\bar{S}_{11} \mathbf{e}_{1} \otimes \mathbf{e}_{1}+\bar{S}_{22} \mathbf{e}_{2} \otimes \mathbf{e}_{2}+\bar{S}_{33} \mathbf{e}_{3} \otimes \mathbf{e}_{3}\right)+A_{2} S_{12}\left(\mathbf{e}_{1} \otimes \mathbf{e}_{2}+\mathbf{e}_{2} \otimes \mathbf{e}_{1}\right) .
$$

In the general three-dimensional theory we have

$$
\operatorname{Curl} \mathbf{K}^{-1}=e_{i k l} G_{j l, k} \mathbf{e}_{i} \otimes \mathbf{e}_{j}
$$

The specialization to the present situation is

$$
\operatorname{Curl} \mathbf{K}^{-1}=\varepsilon_{\beta \alpha} G_{\mu \alpha, \beta} \mathbf{k} \otimes \mathbf{e}_{\mu},
$$

where $\varepsilon_{\beta \alpha}=e_{\beta \alpha 3}$. Equation (72) combines with (25) and $\mathbf{K}^{-1} \mathbf{k}=\mathbf{k}$ to generate the dislocation density

$$
\alpha=\mathbf{k} \otimes \mathbf{a},
$$

corresponding to dislocations of pure edge type, where $\mathbf{a}=a_{\mu} \mathbf{e}_{\mu}$ is the 2-vector defined by

$$
a_{\mu}=J_{K} \varepsilon_{\beta \alpha} G_{\mu \alpha, \beta}
$$

in which $J_{K}=1$ by virtue of the flow rule.

\subsection{Discretization}

The details of the numerical method used in this work have been discussed comprehensively elsewhere

[31] and so we confine ourselves to an outline the method. The spatial discretization of the equations is based on the Green-Stokes theorem

$$
\int_{D} \sigma_{\alpha, \alpha} d a=\varepsilon_{\alpha \beta} \int_{\partial D} \sigma_{\alpha} d x_{\beta},
$$


where $\sigma_{\alpha}\left(x_{1}, x_{2}\right)$ is a smooth two-dimensional vector field, $D$ is an arbitrary simply-connected subregion of $\Omega$ and commas followed by subscripts identify partial derivatives with respect to the coordinates. In this method the plane $\Omega$ is sub-divided into quadrilateral cells. These need not be rectangular, and so it is possible to cover a domain with curved or irregular boundaries with high precision by using a sufficient number of cells.

To approximate the divergence $\sigma_{\alpha, \alpha}$ at node $(i, j)$, the left-hand side of $(75)$ is estimated as the nodal value of the integrand multiplied by the area of $D$; the right-hand side as the mid-point values, labelled with half-integer superscripts, of the integrand on each of the four edges of $\partial D$ multiplied by the appropriate edge length. Thus [31],

$$
\begin{aligned}
2 A^{i, j}\left(\sigma_{\alpha, \alpha}\right)^{i, j}= & \varepsilon_{\alpha \beta}\left[\sigma_{\alpha}^{i+1 / 2, j+1 / 2}\left(x_{\beta}^{i, j+1}-x_{\beta}^{i+1, j}\right)+\sigma_{\alpha}^{i-1 / 2, j+1 / 2}\left(x_{\beta}^{i-1, j}-x_{\beta}^{i, j+1}\right)\right. \\
& \left.+\sigma_{\alpha}^{i-1 / 2, j-1 / 2}\left(x_{\beta}^{i, j-1}-x_{\beta}^{i-1, j}\right)+\sigma_{\alpha}^{i+1 / 2, j-1 / 2}\left(x_{\beta}^{i+1, j}-x_{\beta}^{i, j-1}\right)\right]
\end{aligned}
$$

where

$$
A^{i, j}=\frac{1}{4}\left[\left(x_{2}^{i-1, j}-x_{2}^{i+1, j}\right)\left(x_{1}^{i, j+1}-x_{1}^{i, j-1}\right)-\left(x_{1}^{i-1, j}-x_{1}^{i+1, j}\right)\left(x_{2}^{i, j+1}-x_{2}^{i, j-1}\right)\right]
$$

is one-half the area of the quadrilateral. This is used with $\sigma_{\alpha}=P_{k \alpha} ; k \in\{1,2\}$ to estimate the divergence of the stress at the nodes.

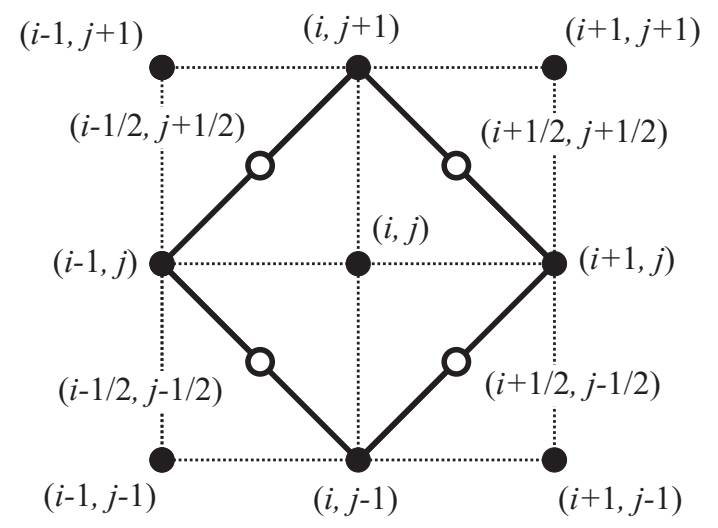

Figure 1: Unit cell of the finite difference mesh.

We also have need of gradients of various functions at zone-centered points. First, we apply (75) with $\sigma_{\alpha}=c_{\alpha} \sigma\left(x_{1}, x_{2}\right)$, where $\sigma$ is a smooth scalar field and $c_{\alpha}$ are arbitrary constants. This yields

$$
\int_{D} \sigma_{, \alpha} d a=\varepsilon_{\alpha \beta} \int_{\partial D} \sigma d x_{\beta}
$$

This is used to estimate the gradient $\sigma_{, \alpha}$ at the half nodes. The four edge contributions to the right-hand side are approximated by replacing the integrand in each with the average of the nodal values at the endpoints. The left-hand side is approximated by the product of the enclosed area with the integrand, evaluated at the half node, yielding [31]

$2 A^{i+1 / 2, j+1 / 2}\left(\sigma_{, \alpha}^{i+1 / 2, j+1 / 2}\right)=\varepsilon_{\alpha \beta}\left[\left(\sigma^{i+1, j+1}-\sigma^{i, j}\right)\left(x_{\beta}^{i, j+1}-x_{\beta}^{i+1, j}\right)-\left(\sigma^{i, j+1}-\sigma^{i+1, j}\right)\left(x_{\beta}^{i+1, j+1}-x_{\beta}^{i, j}\right)\right]$,

where

$$
A^{i+1 / 2, j+1 / 2}=\frac{1}{2}\left[\left(x_{2}^{i, j+1}-x_{2}^{i+1, j}\right)\left(x_{1}^{i+1, j+1}-x_{1}^{i, j}\right)-\left(x_{1}^{i, j+1}-x_{1}^{i+1, j}\right)\left(x_{2}^{i+1, j+1}-x_{2}^{i, j}\right)\right] .
$$


This formula is used with $\sigma=y_{k}, k \in\{1,2\}$ to estimate the deformation gradient at the half nodes, which in turn is required to generate the half-node values of the stress from the relevant constitutive equation.

Eq. (5) is of the form

$$
\sigma_{\alpha, \alpha}=\rho_{r} \ddot{\sigma}
$$

where $\sigma_{\alpha}=P_{k \alpha}$ and $\sigma=y_{k}(k=1,2)$. To represent this at node $(i, j)$ we integrate it over the region enclosed by a quadrilateral with vertices at the nearest-neighbor nodes, obtaining

$$
\Sigma^{i, j, n}=m^{i, j} \ddot{\sigma}^{i, j, n}
$$

where $m^{i, j}=2 A^{i, j} \rho_{r}$ is the nodal mass, $n$ is the time step, superposed dots refer to derivatives with respect to time, and where

$$
\Sigma^{i, j}=2 A^{i, j}\left(\sigma_{\alpha, \alpha}\right)^{i, j}
$$

Here the right-hand side is evaluated in terms of the zone-centered values of $\sigma_{\alpha}$ via (76). The latter are determined constitutively by corresponding zone-centered values of the gradients $\sigma_{, \alpha}$ which, in turn, are given via (79) by the values of $\sigma$ at the nodes located at the vertices of the upper right-hand quadrant of Figure 1. The scheme is seen to require one degree less differentiability than that required by the local differential equations. Traction-free boundaries are simulated by arranging nodes to lie on the boundary and setting to zero the stresses at zone-centered points exterior to the mesh. A discussion of the associated truncation errors is given by Silling [31].

The system (82) is discretized in time using the explicit scheme

$$
\begin{aligned}
m^{i, j} \dot{\sigma}^{i, j, n+1} & =m^{i, j} \dot{\sigma}^{i, j, n}+h \Sigma^{i, j, n} \\
\sigma^{i, j, n+1} & =\sigma^{i, j, n}+h \dot{\sigma}^{i, j, n+1}
\end{aligned}
$$

which is used to advance the solution in time node-by-node. Numerical stability is assured by using sufficiently small time steps selected on the basis of successive trials based on a sequence of values of $h$.

To compute the dislocation density we form the deformation gradient at zone-centered points, and compute the current value of the plastic deformation $\mathbf{G}$ at the same points by forward integration of the flow rule. We then form $\mathbf{H}=\mathbf{F G}^{-1}$ at zone-centered points and construct the associated elastic strain $\mathbf{E}$ and the stress $\mathbf{S}$. The flow rule with dislocation-based hardening requires the dislocation vector a at zone-centered points. To obtain this we fix $\mu \in\{1,2\}$ and use (75) with $\sigma_{\alpha}$ replaced by

$$
\sigma_{\mu \alpha}=\varepsilon_{\alpha \gamma} G_{\mu \gamma} ; \quad a_{\mu}=\sigma_{\mu \alpha, \alpha}
$$

Then the nodal values of $a_{\mu}$ are obtained from (76), which may be simplified by using $\varepsilon_{\alpha \beta} \varepsilon_{\alpha \gamma}=\delta_{\beta \gamma}$. The zone-centered values, required in (44), (53), (54) and (73), where $|\alpha|=|\mathbf{a}|$, are obtained by averaging the values at adjacent nodes.

We confine attention to rate-dependent response and integrate the flow rule (44) using the scheme $\sigma_{\alpha}^{i, j, n+1}=\sigma_{\alpha}^{i, j, n}+h \dot{\sigma}_{\alpha}^{i, j, n}$ at each zone-centered point. We then check inequality $(44)_{2}$ at each time step and decide whether or not to update the plastic deformation accordingly. 


\subsection{Examples}

The problem considered is the in-plane shearing of a block, $2 \mathrm{~mm}$ on a side, of uniform initial mass density $\rho_{r}=7000 \mathrm{~kg} / \mathrm{m}^{3}$. The elastic moduli used in (67) and (68) are (in units of $G P a$ ): $C_{1}=169$, $C_{2}=86$ and $C_{3}=232$. These have been correlated with data on the Voigt moduli for BCC iron. We assume quiescent initial conditions (zero initial displacement and velocity) and impose zero tractions on the upper and lower horizontal edges. The left-side vertical edge is fixed and the right-hand edge is displaced vertically at the rate of $0.4 \mathrm{~m} / \mathrm{s}$. The horizontal velocities of these edges are zero. In the yield function (49), (53) and (54) we use the dimensionless parameters $A_{1}=0.3955, A_{2}=0.4631$ and $p=0.1$, together with the squared yield strength $k_{0}=0.1057(G P a)^{2}$. The plastic spin constants, in units of $(G p a)^{-2}$, are: $B_{0}=1.068, B_{1}=-1.912, B_{2}=30.384$ and $B_{3}=5.3174$.

These and other parameters discussed thus far are associated with a best-fit simulation [13] of homogeneous deformations by the standard of the conventional crystal plasticity theory based on (1) and (55). Here, however, all simulations account for inertia and viscoplasticity with viscosity $\nu^{-10^{-4}}$ $G p a-$ sec. Convergence of the spatial features of the predicted response was achieved using a $48 \times 48$ mesh and a time step of $5 \times 10^{-10} \mathrm{sec}$, which is significantly less than the estimated critical step of $7.35 \times 10^{-9}$ sec for the explicit time integration.

Figures 2 and 3 depict the effect of adjusting the length-scale parameter $k_{1}$ (in units of $m^{2}$ ) in the hardening rule (54). All the frames correspond to a fixed time lapse from the initial instant. The length-scale effect in this rule becomes significant when $k_{1}|\alpha|^{2}$ is of order unity, corresponding to spatial variations in plastic deformation over distances of order $\sqrt{k_{1}}$. The figure suggests a significant interaction between this scale and the predicted spatial variations in plastic deformation. We draw particular attention to the predicted emergence of curved bands of high dislocation intensity interacting with the boundaries of the specimen. These features, reminiscent of pinned dislocation loops, are modulated to some degree by local and global wave interactions associated with inertial effects. The initial value of plastic deformation is taken to be the uniform two-dimensional rotation associated with a lattice inclined at angle $\theta$ to the horizontal. This initial condition is associated with a state of vanishing residual stress [32].

The effect of lattice orientation, associated with adjustment of the lattice inclination angle $\theta$, is shown in Figures 4 and 5, using a single value of the length-scale parameter $k_{1}$. This illustrates the significant effect of the orientation of the specified undistorted lattice relative to the boundaries of the domain.

Due to the paucity of relevant data, we are not able to provide independent corroboration of the these predictions. However, our hope is that the capability of this model to capture length-scale effects and other aspects of crystal plasticity may provide impetus for an experimental program aimed at assessing its predictive potential and quantifying the relatively small number of associated constitutive parameters. In particular, we emphasize the fact that we have considered only a very special case of the broad framework described in Sections 3-5, and so considerable latitude remains for refinement of the model vis à vis quantitative predictions. 
Not shown here are predictions of the transient elastic strain [13]. We find the maximum value to be on the order of two percent, which suggests that relaxation of the assumption of small elastic strain may prove necessary in applications. In particular, while the elastic strain is bounded by the diameter of the elastic range in the rate-independent theory, there is no such limitation in the viscoplastic theory. 


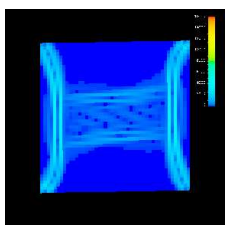

(a) $k=1 \cdot 10^{-10}$

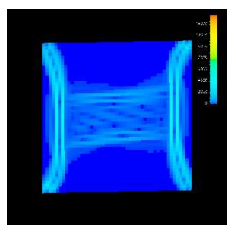

(b) $k=1 \cdot 10^{-8}$

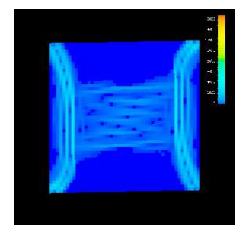

(c) $k=1 \cdot 10^{-6}$

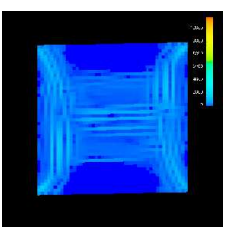

(d) $k=1 \cdot 10^{-4}$

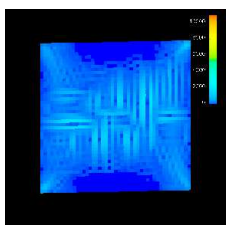

(e) $k=1 \cdot 10^{-2}$

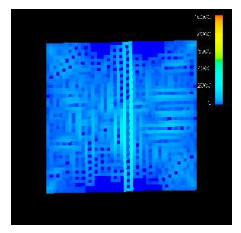

(f) $k=1$

Figure 2: Spatial variation of $|\boldsymbol{\alpha}| . \theta=10^{\circ}, \mathrm{t}=10 \mu \mathrm{s} . k_{1}$ is given in units of $\mathrm{m}^{2}$.

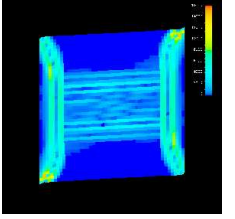

(a) $k=1 \cdot 10^{-10}$

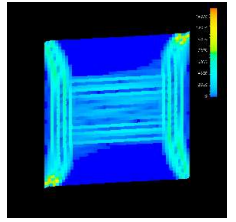

(b) $k=1 \cdot 10^{-8}$

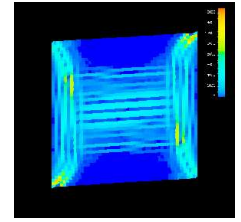

(c) $k=1 \cdot 10^{-6}$

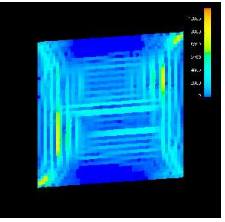

(d) $k=1 \cdot 10^{-4}$

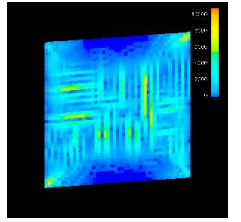

(e) $k=1 \cdot 10^{-2}$

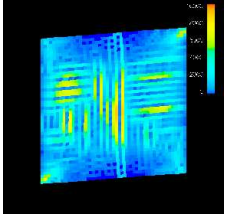

(f) $k=1$

Figure 3: Spatial variation of $|\boldsymbol{\alpha}| . \theta=10^{\circ}, \mathrm{t}=40 \mu s . k_{1}$ is given in units of $m^{2}$.

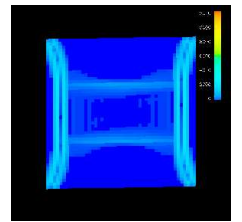

(a) $\theta=0$

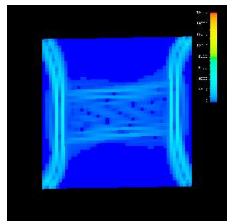

(b) $\theta=10$

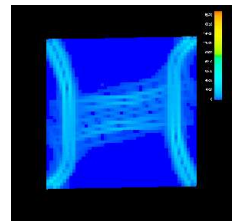

(c) $\theta=20$

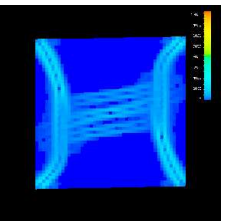

(d) $\theta=30$

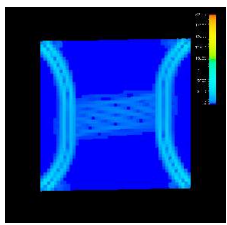

(e) $\theta=40$

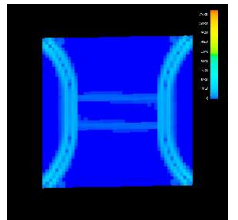

(f) $\theta=45$

Figure 4: Lattice orientation $(\theta)$ dependence. The figures depict the spatial variation of $|\boldsymbol{\alpha}| . \quad k_{1}=$ $1 \cdot 10^{-10} m^{2} \cdot t=10 \mu s$.

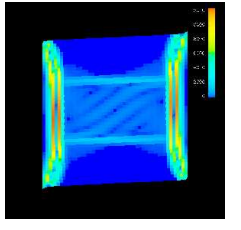

(a) $\theta=0$

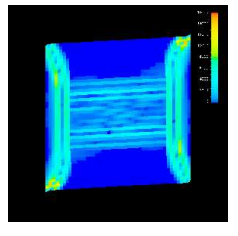

(b) $\theta=10$

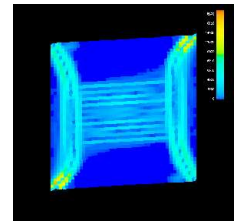

(c) $\theta=20$

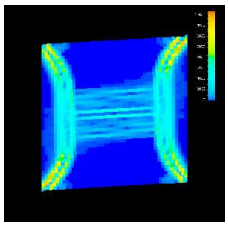

(d) $\theta=30$

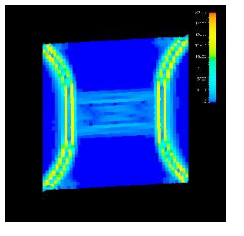

(e) $\theta=40$

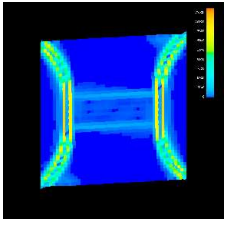

(f) $\theta=45$

Figure 5: Lattice orientation $(\theta)$ dependence. The figures depict the spatial variation of $|\boldsymbol{\alpha}| . \quad k_{1}=$ $1 \cdot 10^{-10} m^{2} \cdot t=40 \mu s$. 


\section{References}

1. K.S. Havner, 1992, Finite Plastic Deformation of Crystalline Solids. Cambridge University Press.

2. M.E. Gurtin, E. Fried and L. Anand, 2010, Mechanics and Thermodynamics of Continua. Cambridge University Press.

3. S. Ha and K. T. Kim, 2011, Heterogeneous deformation of Al single crystal: Experiments and finite element analysis. Math. Mech. Solids 16, 652-661.

4. V.A. Lubarda, 2002, Elastoplasticity Theory. CRC Press, Boca Raton, FL.

5. G. Rengarajan and K. Rajagopal, 2001, On the form for the plastic velocity gradient $L_{p}$ in crystal plasticity. Math. Mech. Solids 6, 471-80.

6. L. Deseri and D.R. Owen, 2002, Invertible structured deformations and the geometry of multiple slip in single crystals. Int. J. Plasticity 18, 833-849.

7. G. Del Piero and D.R. Owen, 1993, Structured deformations of continua. Arch. Rat. Mech. Anal. 124, 99-155.

8. D. Owen, private e-mail communication dated June 6, 2012.

9. 11. P.M. Nagdhi, 1990, A critical review of the state of finite-strain plasticity. ZAMP 41, 315-394.

10. P.M. Naghdi and A. Srinivasa, 1994, Some general results in the theory of crystallographic slip. ZAMP 45, 687-732.

11. P.M. Nagdhi and Srinivasa, 1994, Characterization of dislocations and their influence on plastic deformation in single crystals. Int. J. Engng. Sci. 32, 1157-1182.

12. J.F. Bell and R.E. Green, Jr., 1967, An experimental study of the double-slip deformation hypothesis for face-centered cubic crystals. Phil. Mag. 15, 469-476.

13. J. Edmiston, 2012, Continuum plasticity: Phenomenological modeling and x-ray diffraction experiments. Dissertation. UC Berkeley.

14. S. Cleja-Tigoiu and E. Soos, 1990, Elastoviscoplastic models with relaxed configurations and internal variables. Appl. Mech. Reviews 43, 131-51.

15. M. Epstein and M. Elzanowski, 2007, Material Inhomogeneities and their Evolution. Springer, Berlin.

16. K. Rajagopal and A. Srinivasa, 1998, Inelastic behavious of materials. Part 1: Theoretical underpinnings. Int. J. Plasticity 14, 945

17. A. Gupta, D.J. Steigmann and J.S. Stölken, 2007, On the evolution of plasticity and incompatibility. Math. Mech. Solids 12, 583-610.

18. A. Gupta, D.J. Steigmann and J.S. Stölken, 2011, Aspects of the phenomenological theory of elastic-plastic deformation. J. Elasticity 104, 249-266.

19. D.J. Steigmann and A. Gupta, 2011, Mechanically equivalent elastic-plastic deformations and the problem of plastic spin. Theoretical and Applied Mechanics 38, 397-417.

20. A.E. Green and J.E. Adkins, 1970, Large Elastic Deformations. Oxford University Press.

21. B. Cullity, 1978, Elements of X-Ray Diffraction. Addison-Wesley.

22. W. Prager, 1961, Introduction to the Mechanics of Continua. Ginn \& Co., Boston. 
23. P. Cermelli and M.E. Gurtin, 2001, On the characterization of geometrically necessary dislocations in finite plasticity. J. Mech. Phys. Solids 49, 1539-68.

24. W. Noll, 1967, Materially uniform simple bodies with inhomogeneities. Arch. Ration. Mech. Anal. 27, 1-32.

25. K. Rajagopal and A. Srinivasa, 2005, On the role of the Eshelby energy-momentum tensor in materials with multiple natural configurations. Math. Mech. Solids 10, 3.

26. M. Lucchesi and M. Silhavy, 1991, Il'yushin's conditions in non-isothermal plasticity. Arch. Ration. Mech. Anal. 113, 121-63.

27. W.I. Zangwill, 1969, Nonlinear Programming. Prentice-Hall, Englewood Cliffs, N.J.

28. G.F. Smith, M.M. Smith and R.S. Rivlin, 1963, Integrity bases for a symmetric tensor and a vector - the crystal classes. Arch. Ration. Mech. Anal. 12, 93-133. Reprinted in: Collected Works of R.S. Rivlin, Vol. 1 (G.I. Barenblatt and D.D. Joseph, eds.). Springer, N.Y., 1997.

29. L. Anand, S. Balasubramanian, and M. Kothari, 1997, Constitutive modeling of polycrystalline metals at large strains. In: Large plastic deformation of crystalline aggregates. Springer-Berlin.

30. A.C. Pipkin and R.S. Rivlin, 1959, The formulation of constitutive equations in continuum physics (I). Arch. Rational Mech. Anal. 2, 129-144. Reprinted in: Collected Works of R.S. Rivlin, Vol. 1 (G.I. Barenblatt and D.D. Joseph, eds.). Springer, N.Y., 1997.

31. S.A. Silling, 1988, Finite difference modelling of phase changes and localization in elasticity. Comp. Meth. Appl. Mech. Engng. 70, 251-273.

32. D.J. Steigmann and R.W. Ogden, 2012, Note on residual stress, lattice orientation and dislocation density in crystalline solids. J. Elasticity (DOI 10.1007/s10659-012-9378-1). 
Figure $2 a$
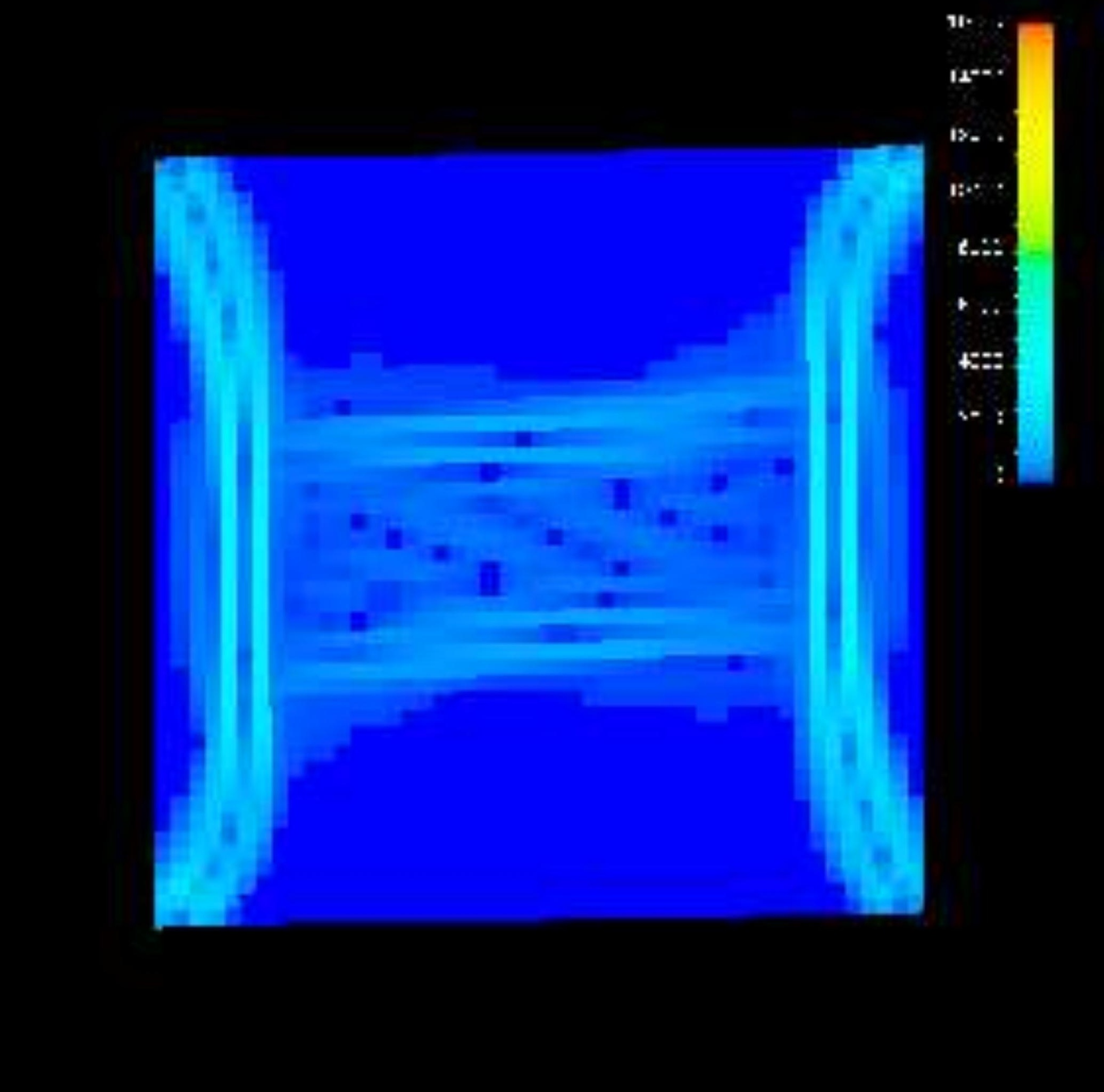

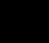

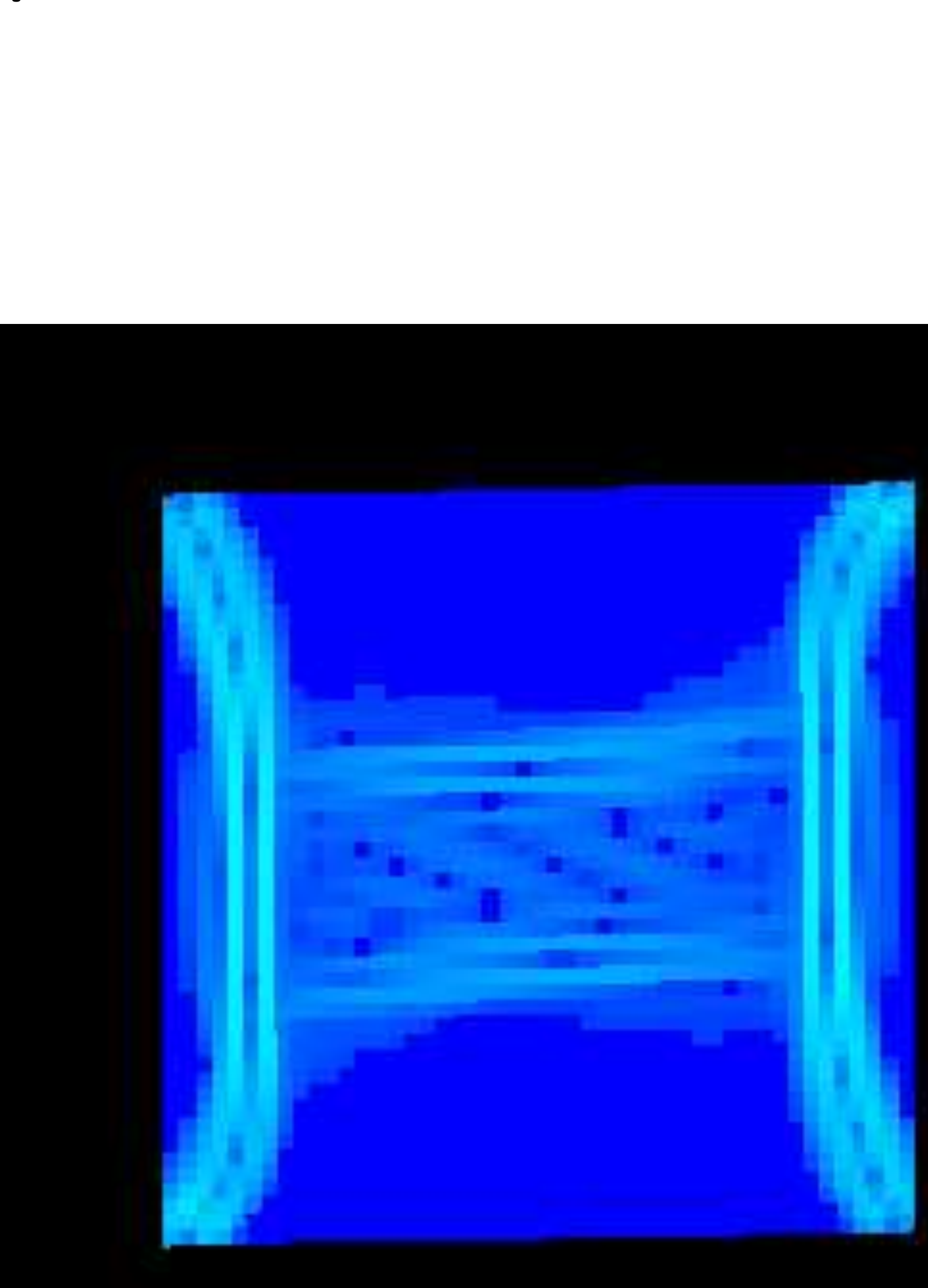
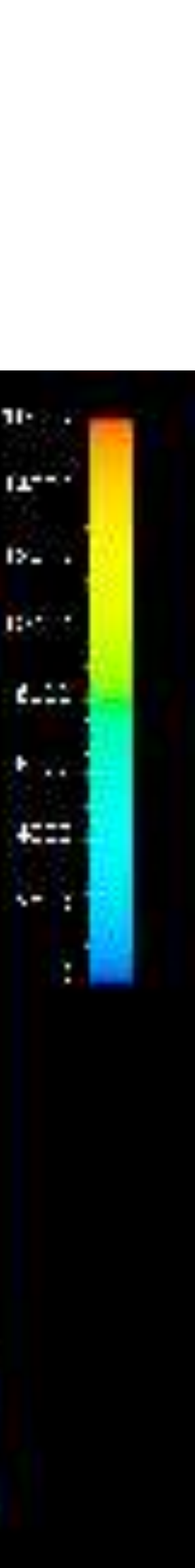
Figure $2 b$
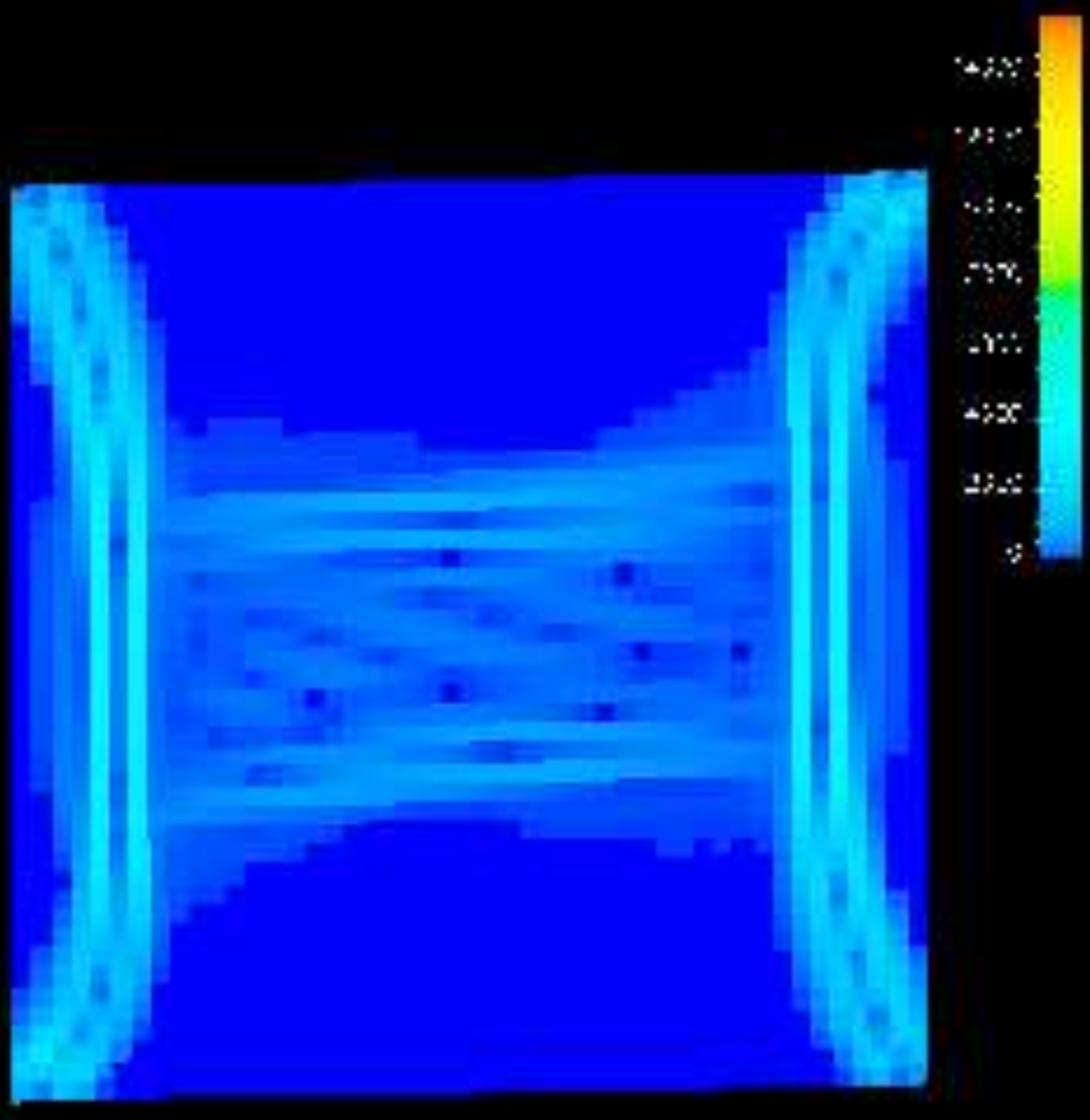

$$
\text { ( }
$$

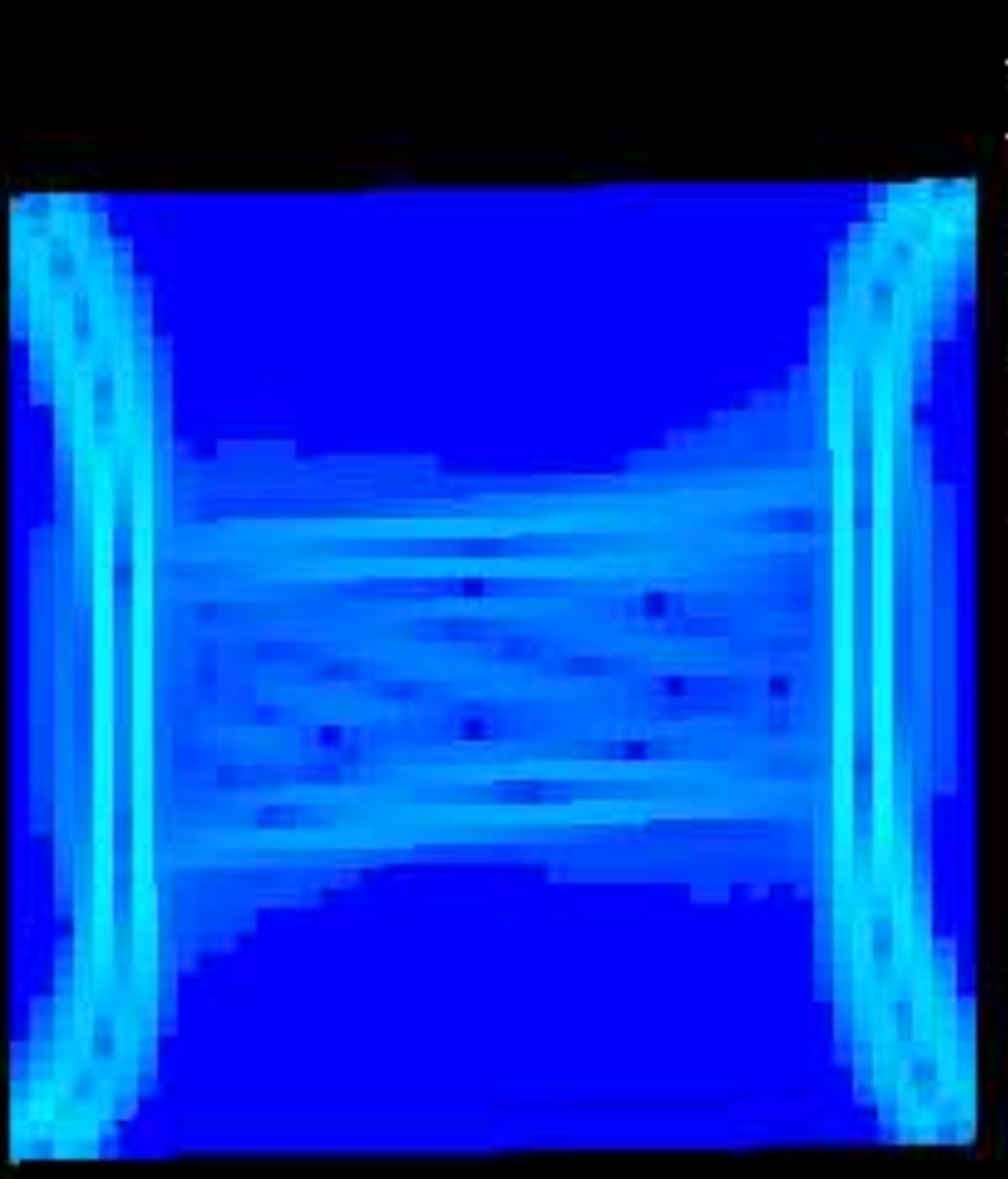

gure 2b

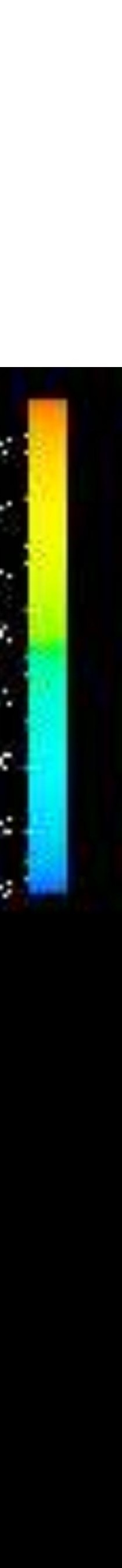


Figure 2c
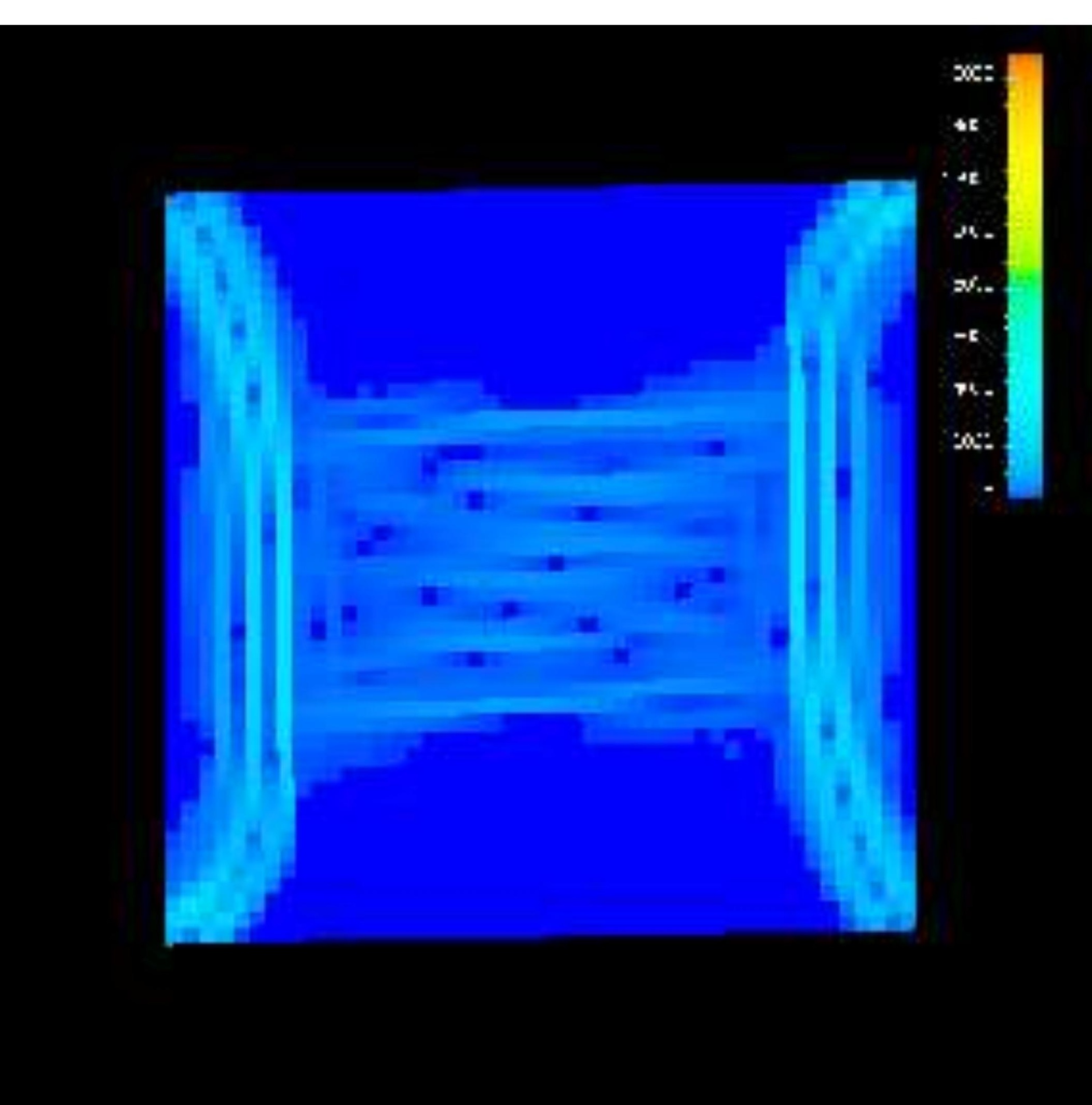


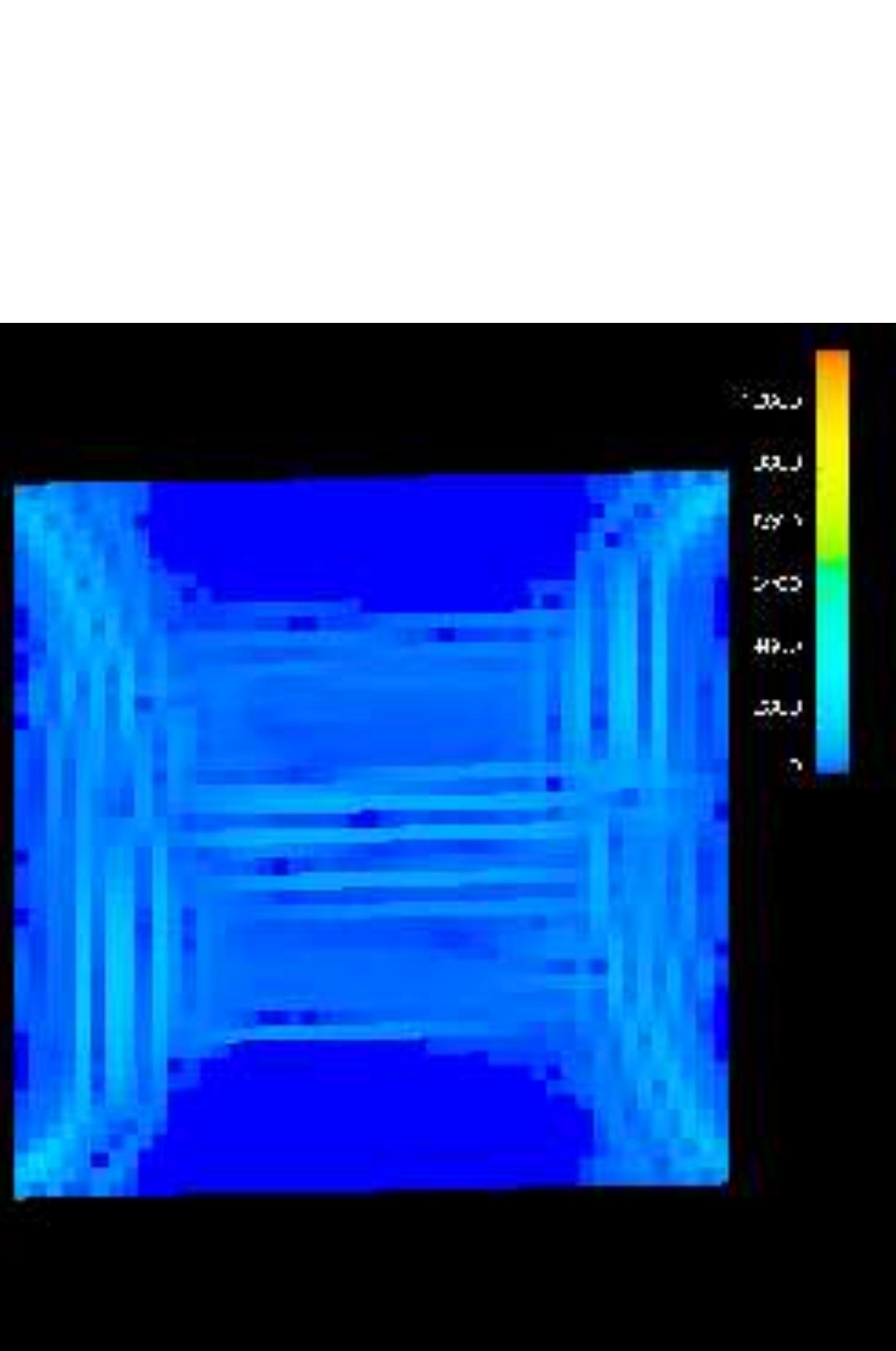

Figure 2d $2 d$

\author{
Figure
}
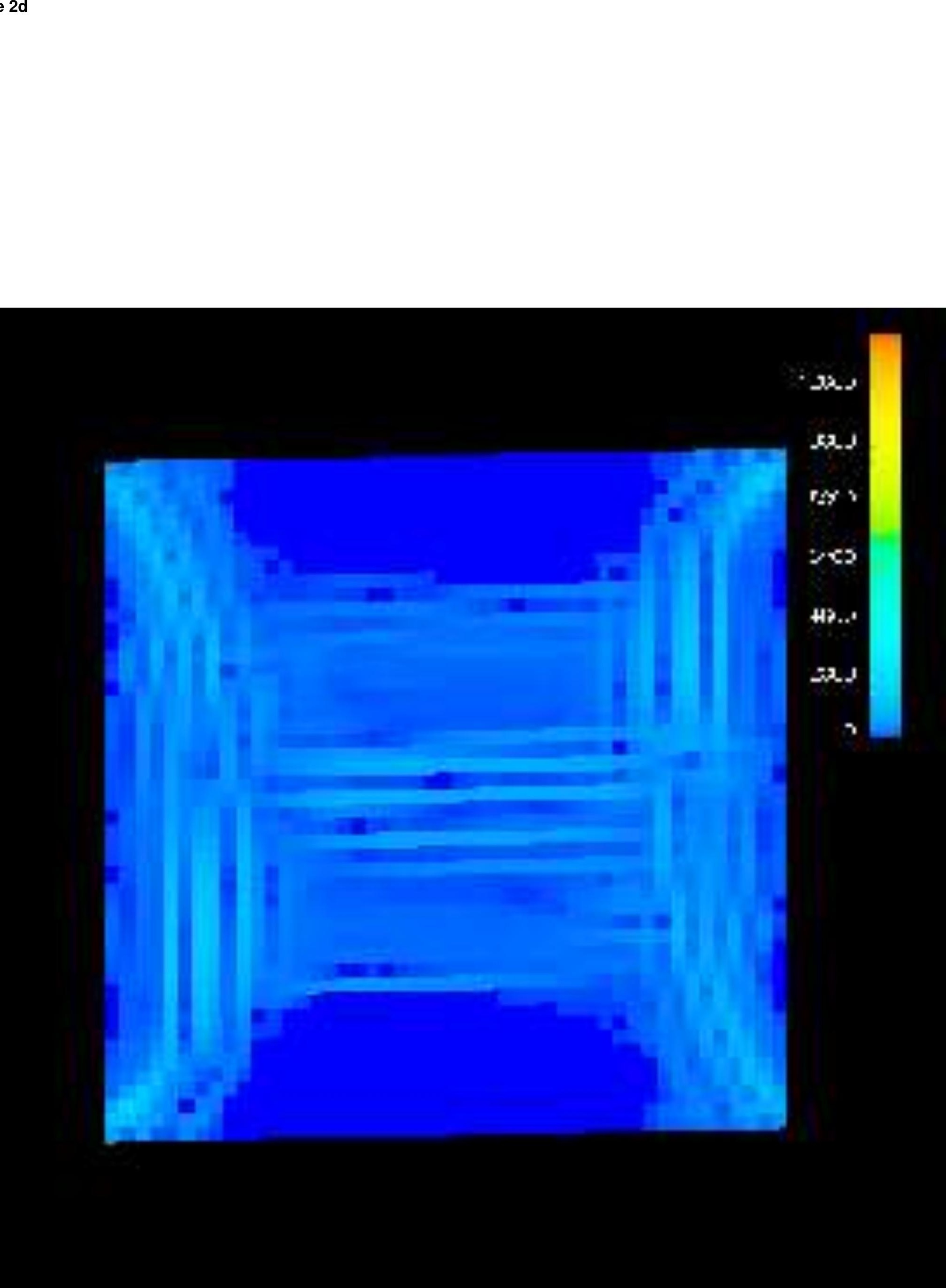
Figure $2 e$

$1: x$ is

$\because 2,14$

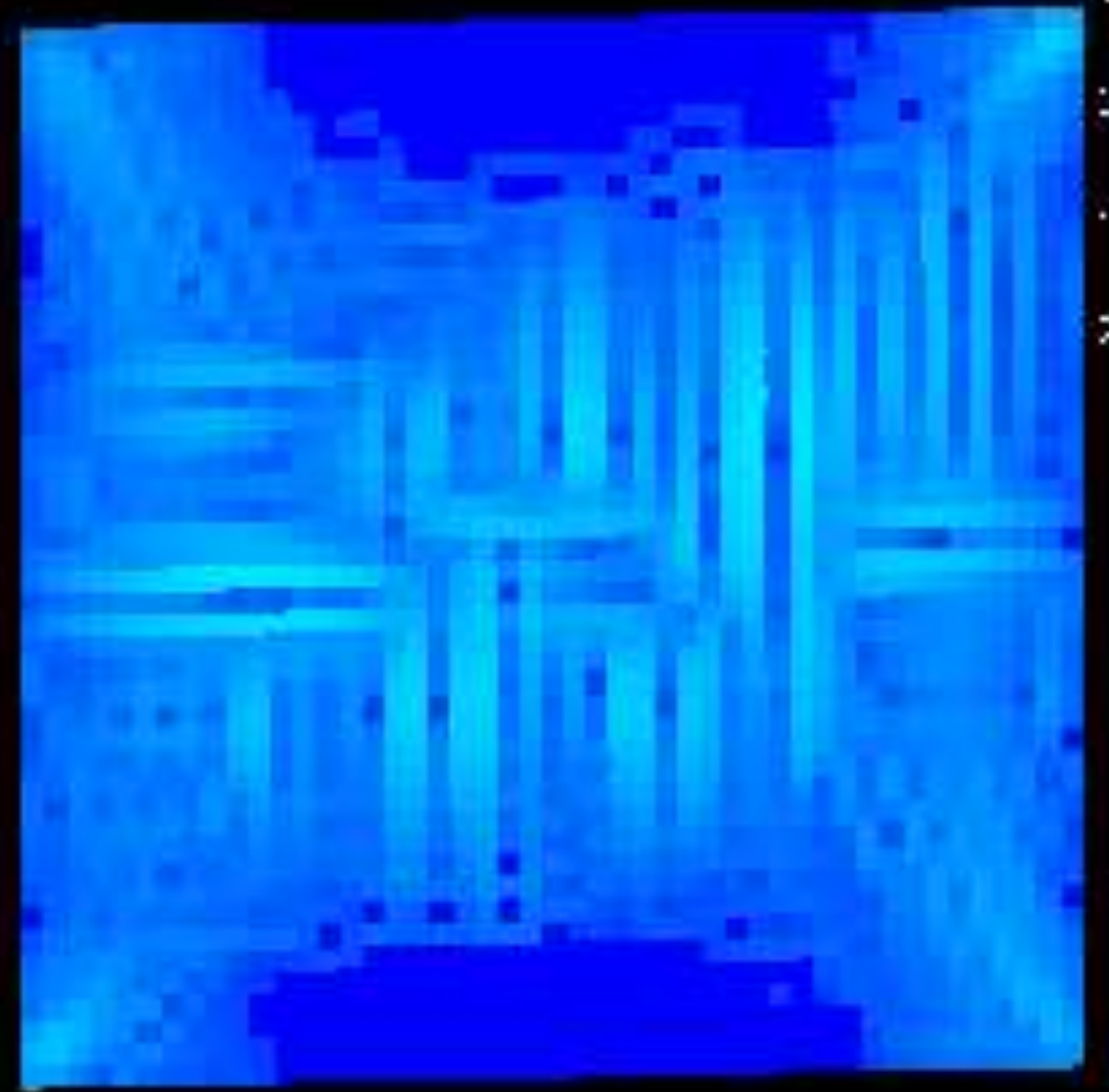

1.

** - 4 .

* *1\%"

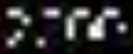




\section{Figure $2 f$}

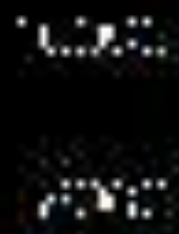
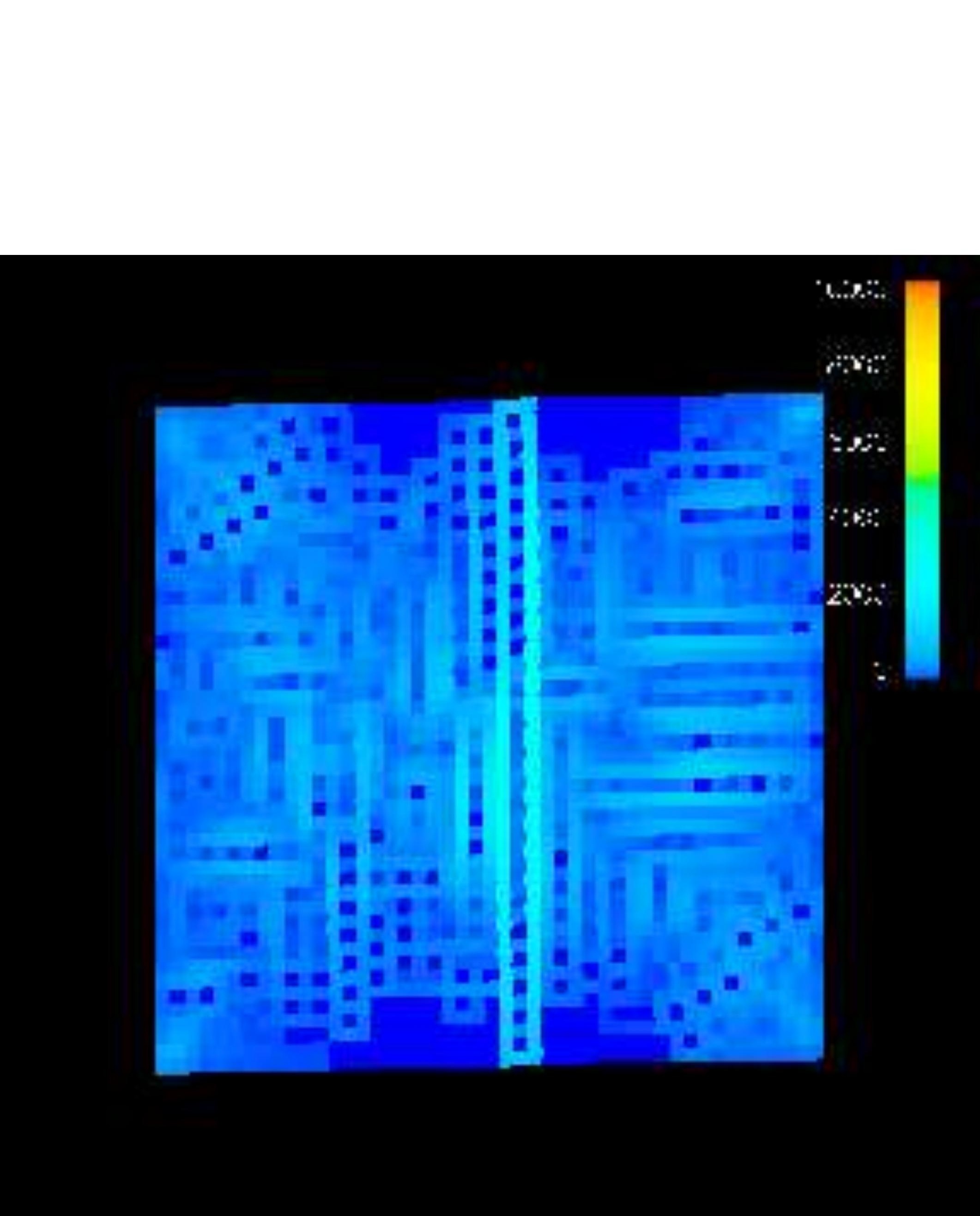

Eure 2$$
\text { (1) }
$$

$f$


Figure $3 a$
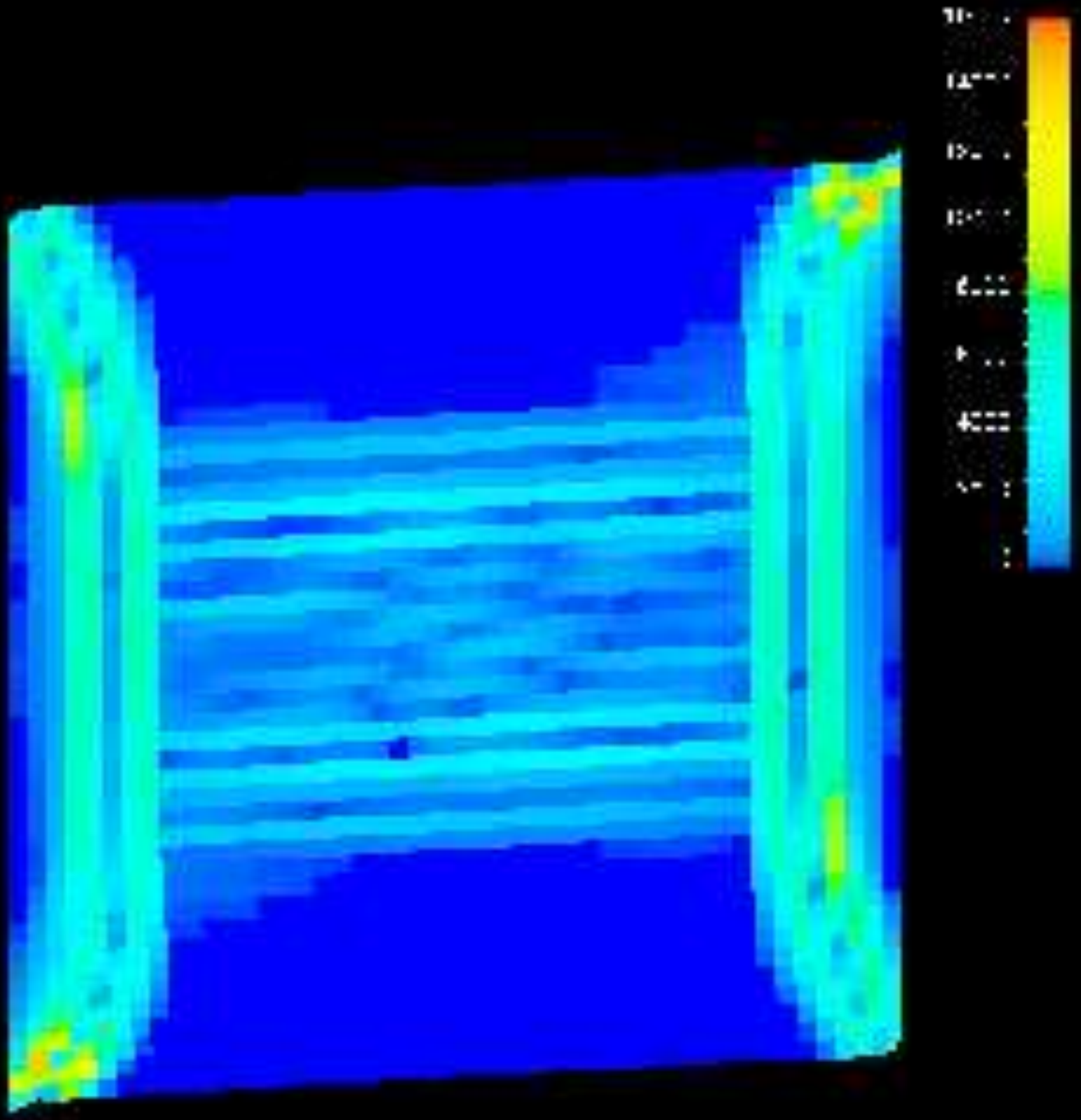


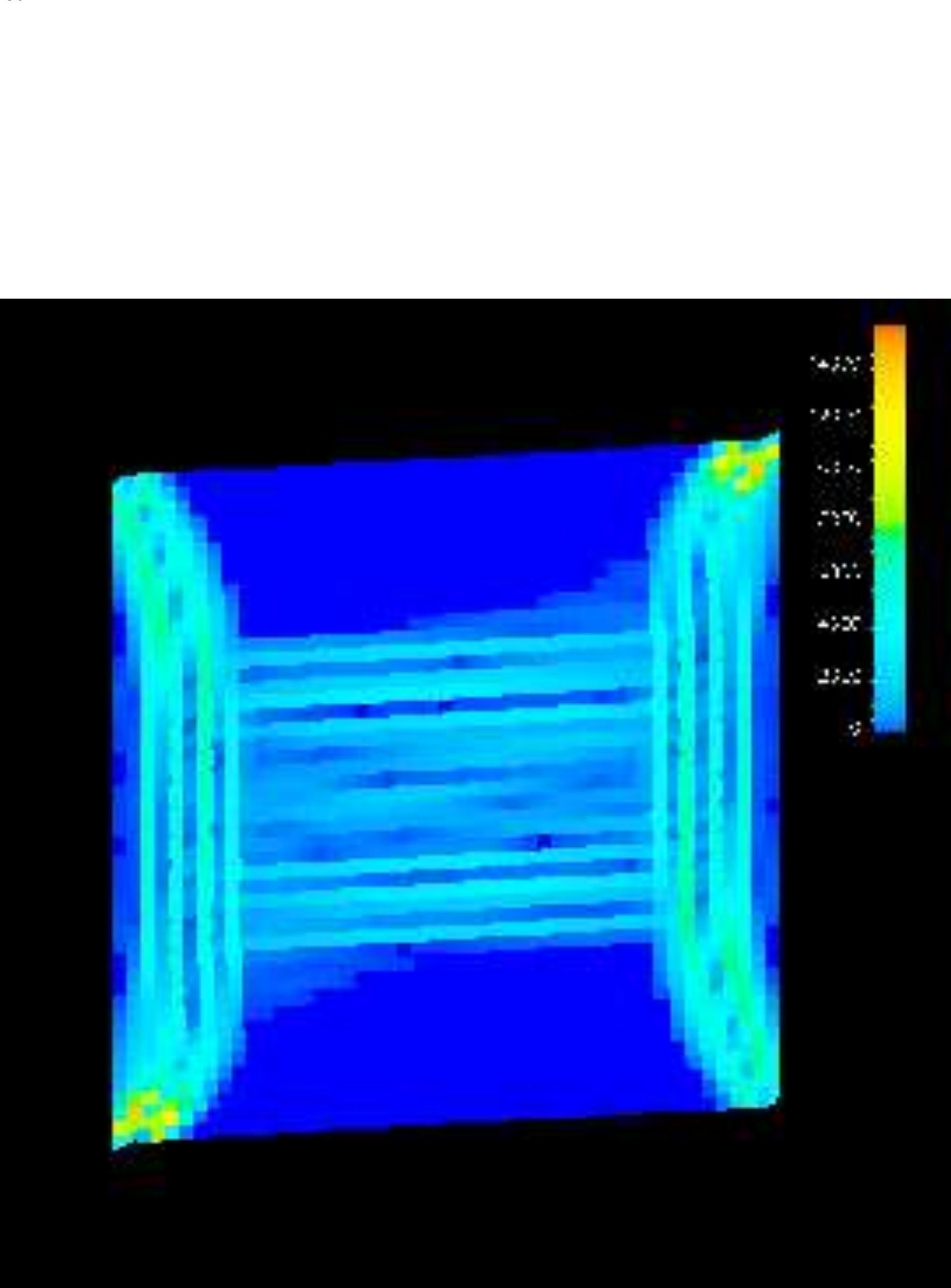

\section{Figure 3} b

b




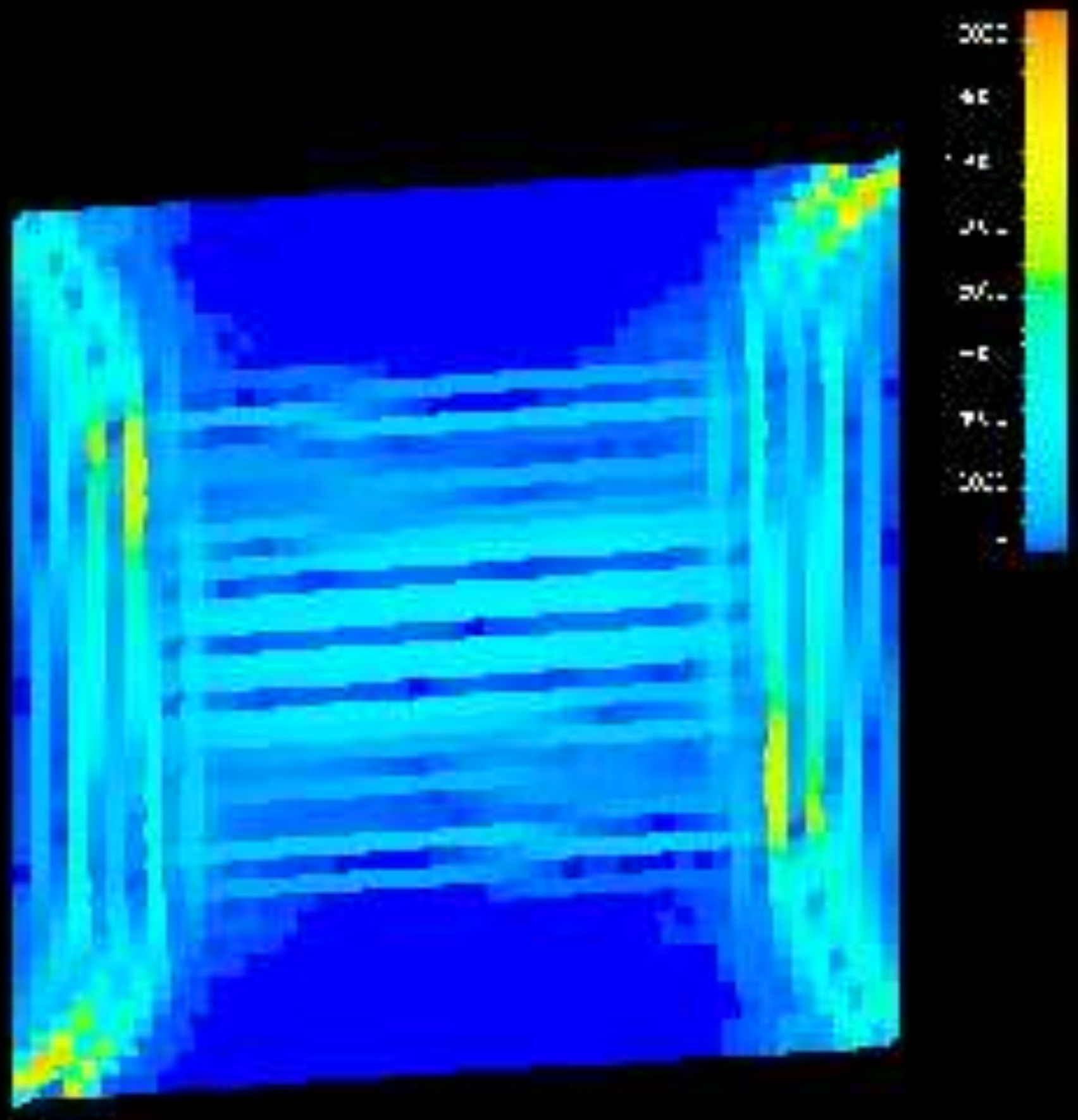

\section{$w=$ \\ $4 \mathbf{5}$}

* $4 \mathrm{E}$

$24=$

sit.

$-\mathbf{r}$

Th.

$20 *$

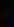


Figure 3d

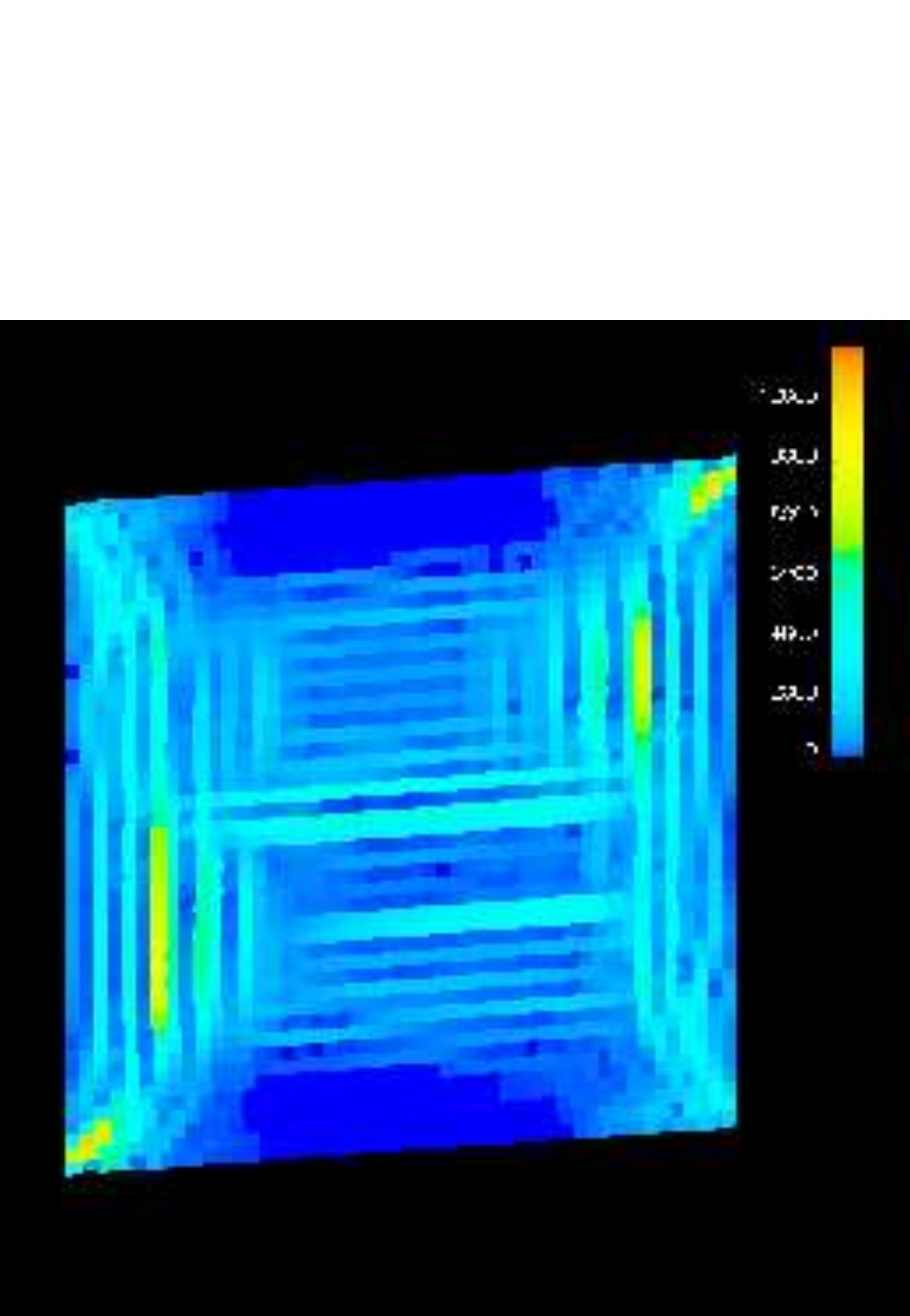

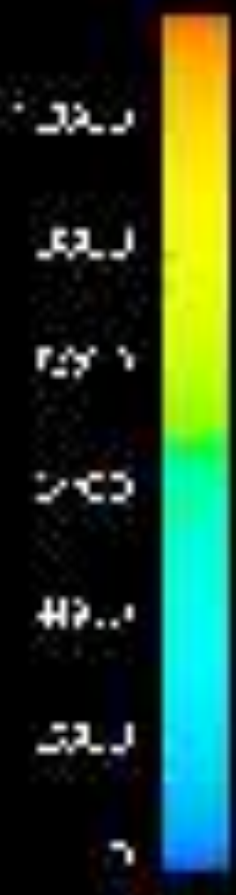

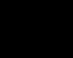
d

\author{
Fure 3
}


Figure $3 e$

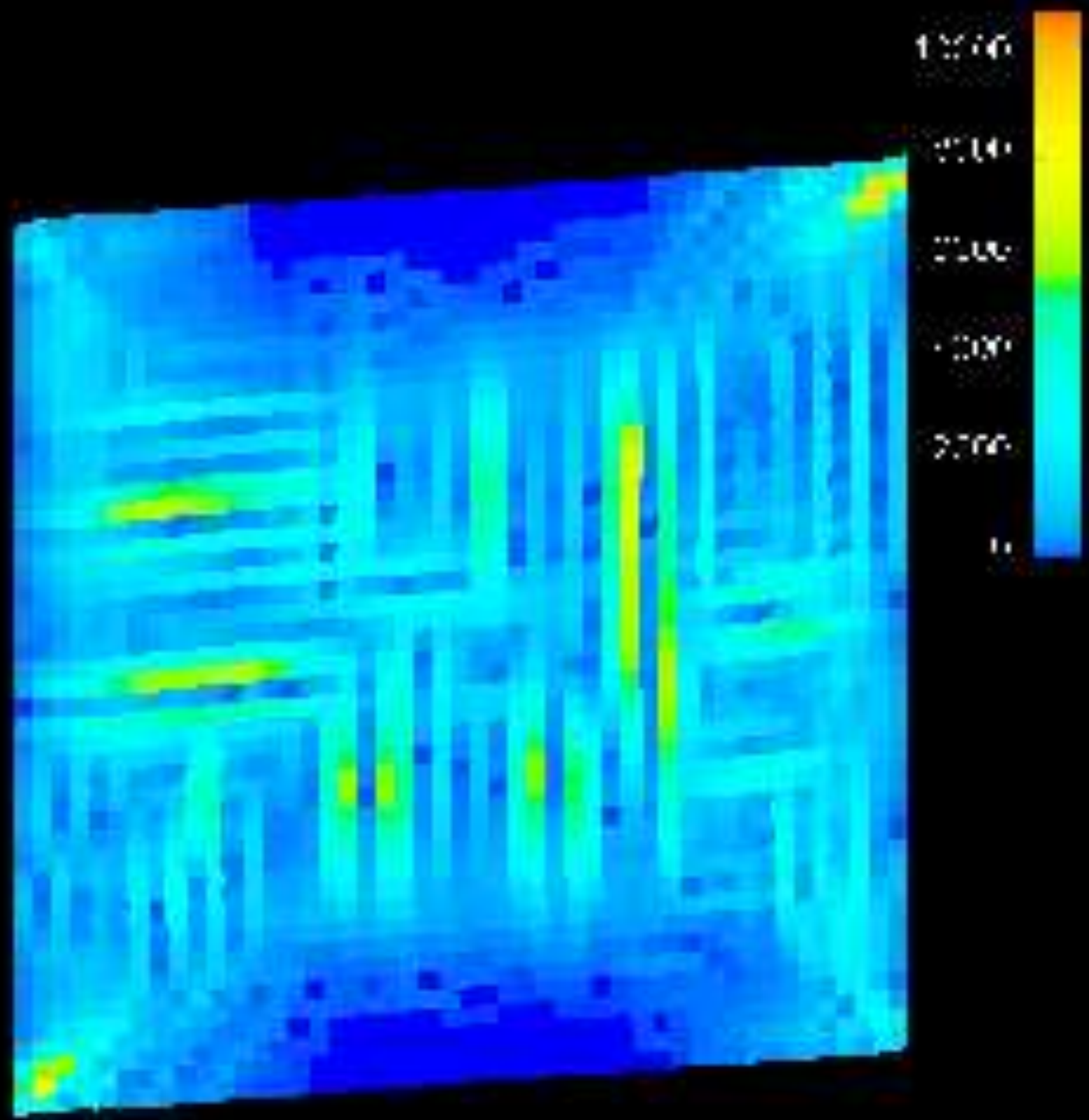

1::ir

$\div 14$

**18.

".t\%"

For.

1. 


\section{Figure $3 f$

\section{"...:.". it:.}

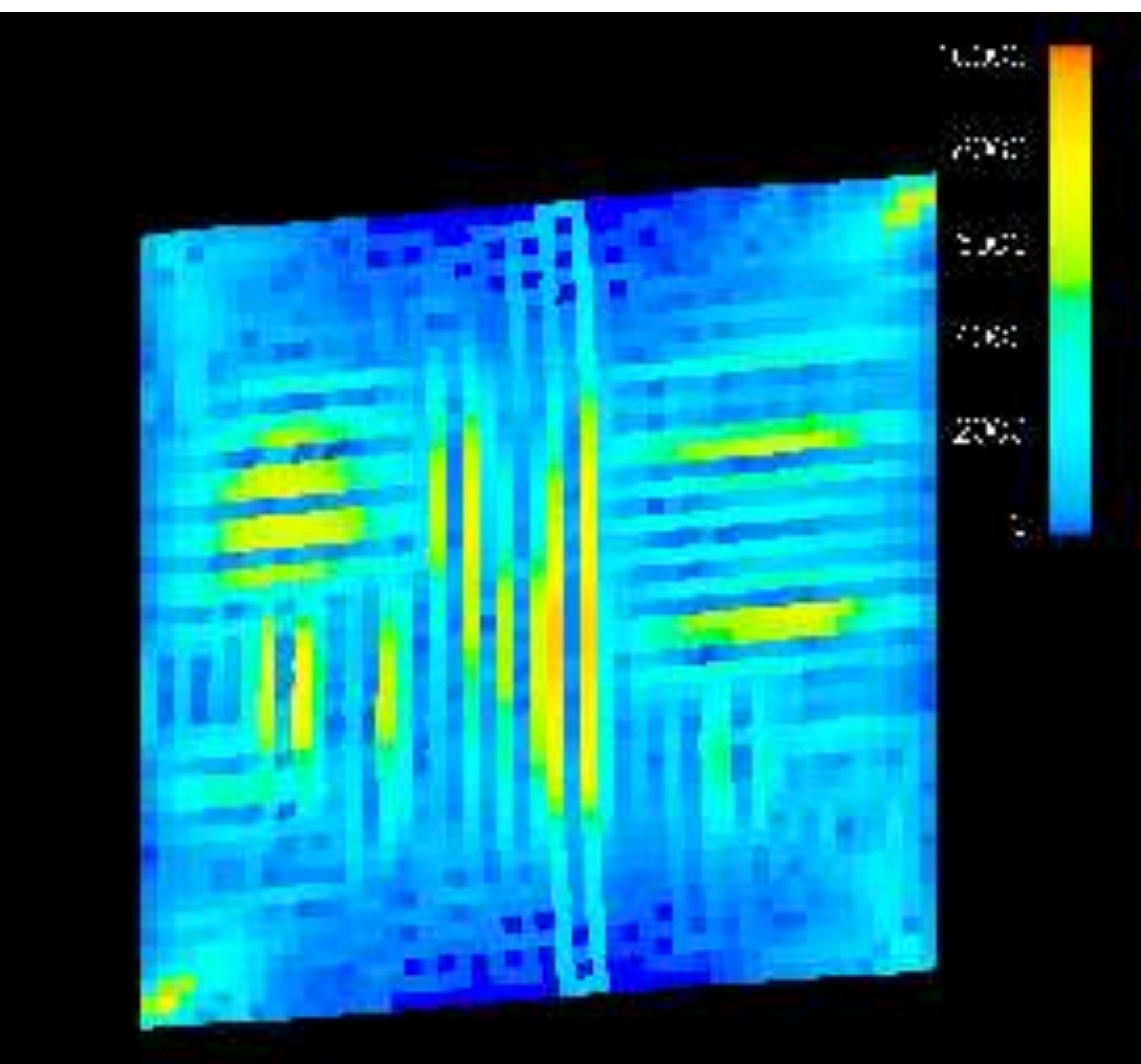

f

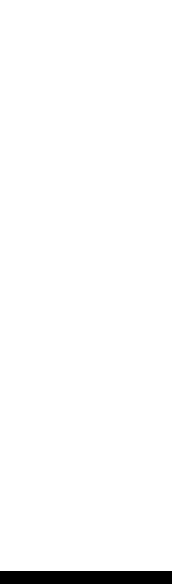




$$
\begin{aligned}
& \therefore \text { I. } \\
& \text {; } \\
& \approx * \mathbf{v} \\
& c: 06 \\
& \text {-:: : } \\
& 48 \\
& \text { : }
\end{aligned}
$$

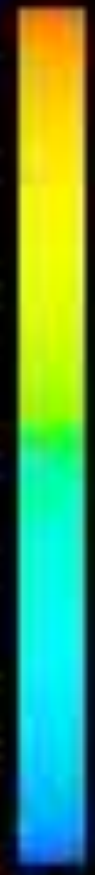



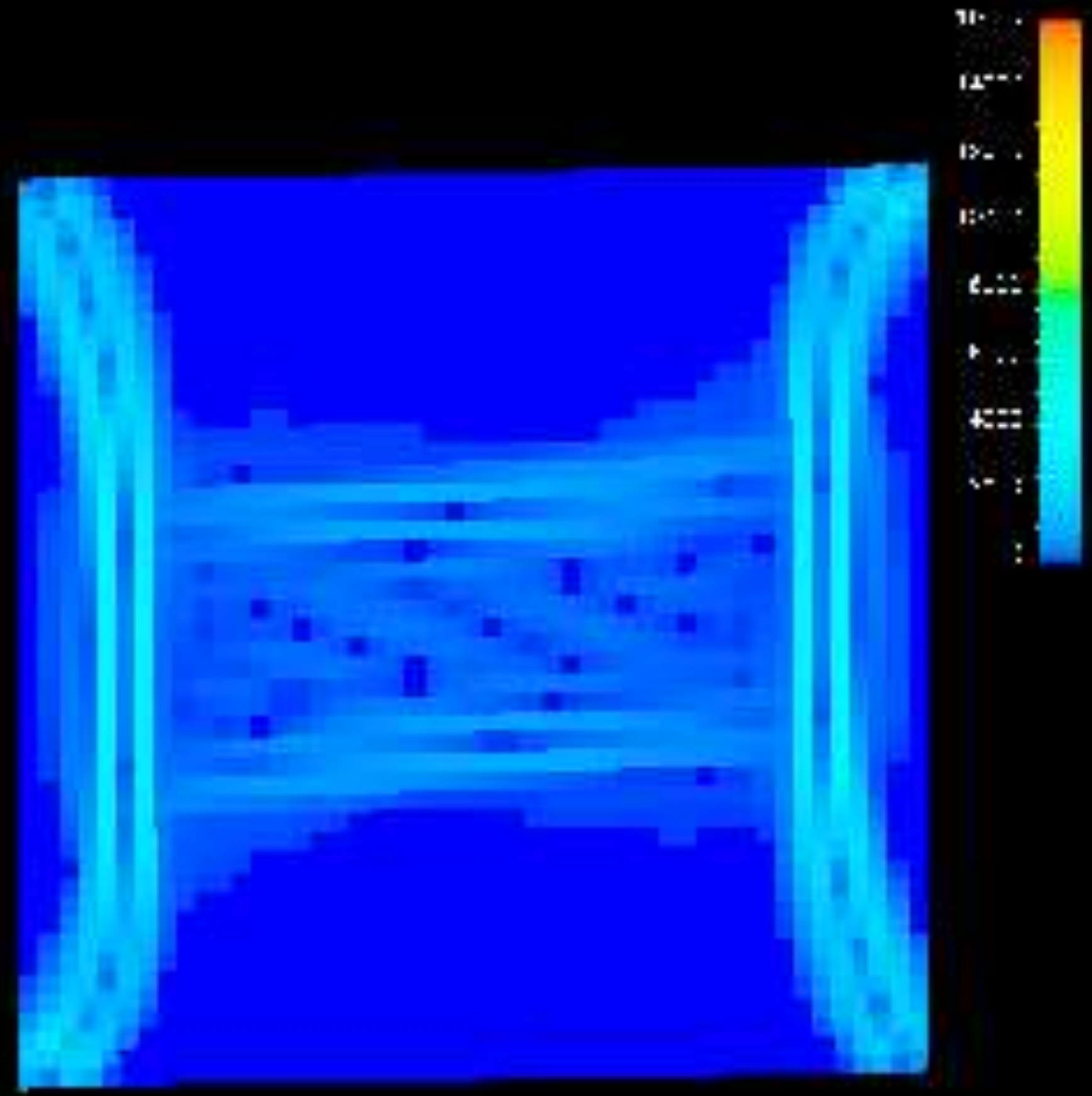

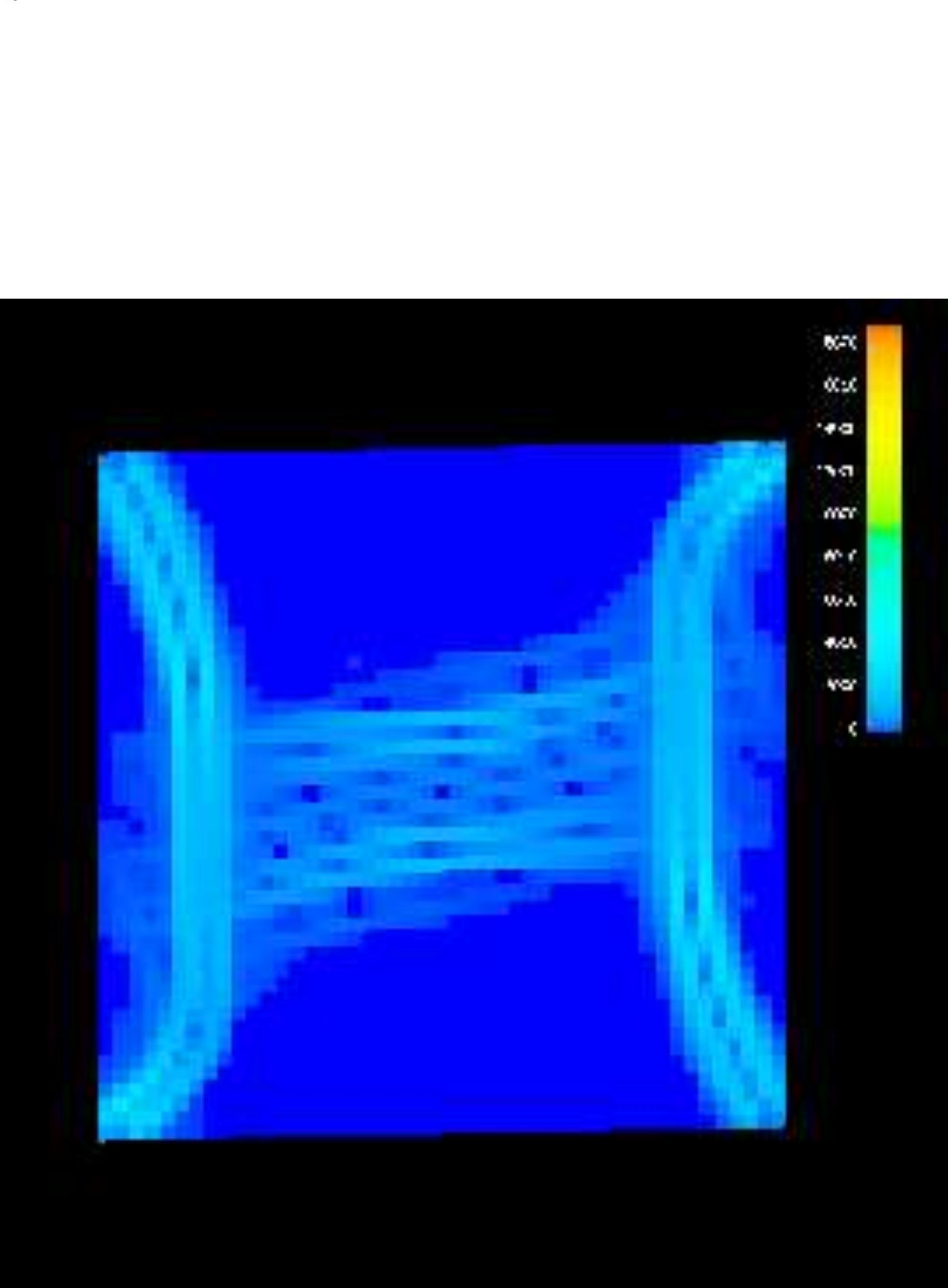

Figure 4c

igure 4c c

c

(

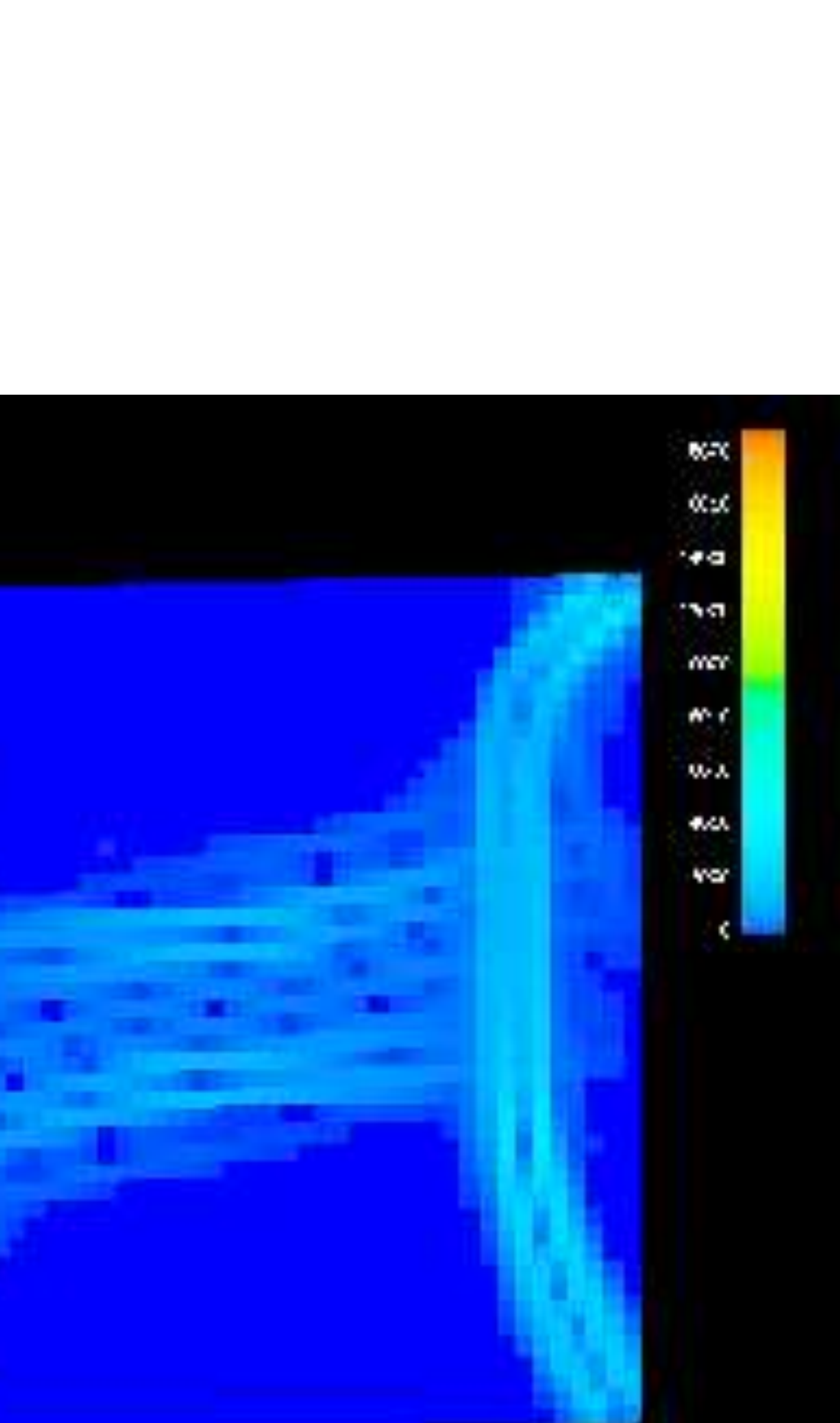



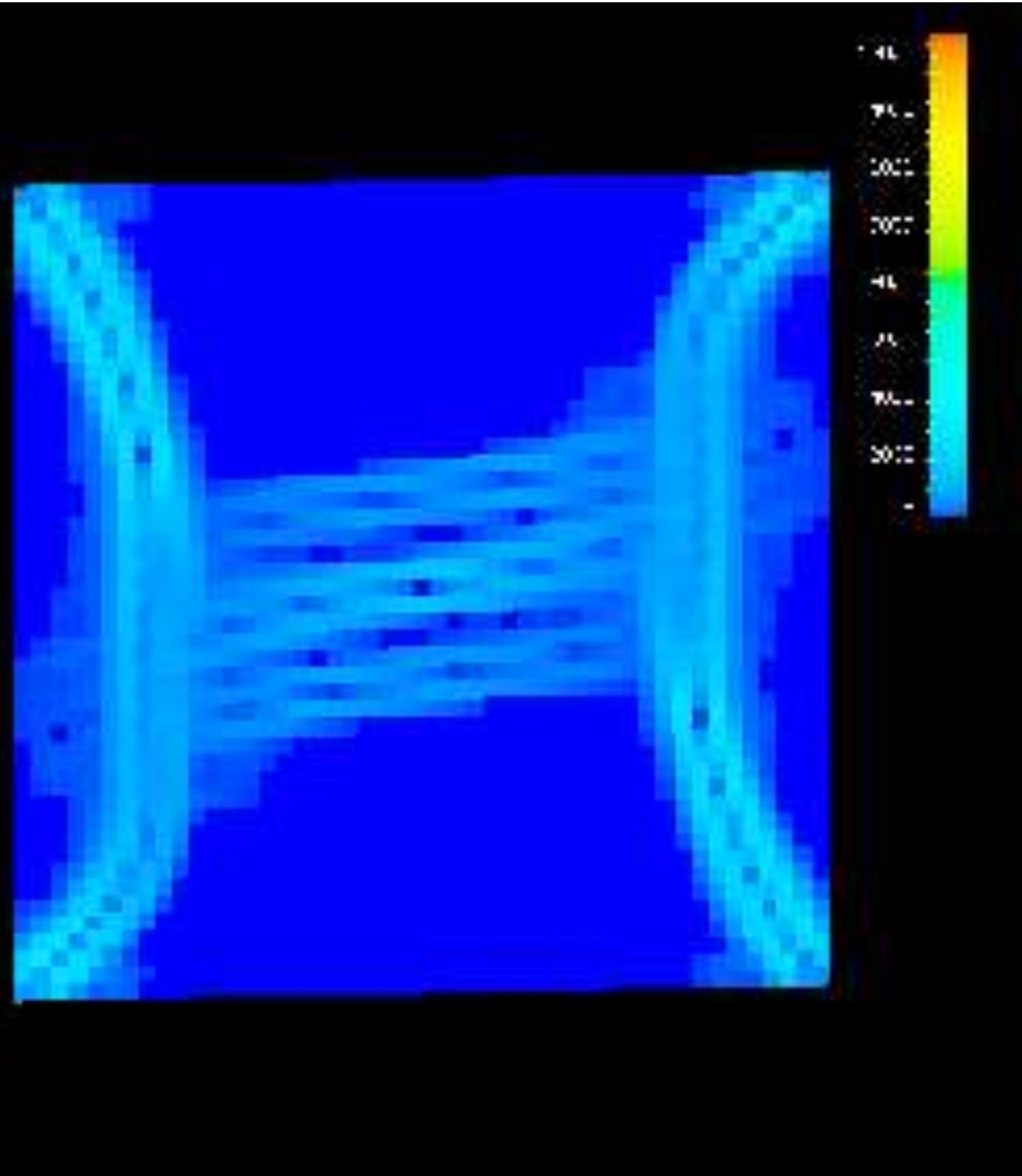

Figure 4d

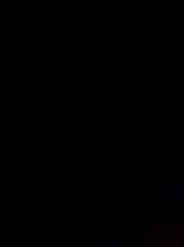
d 

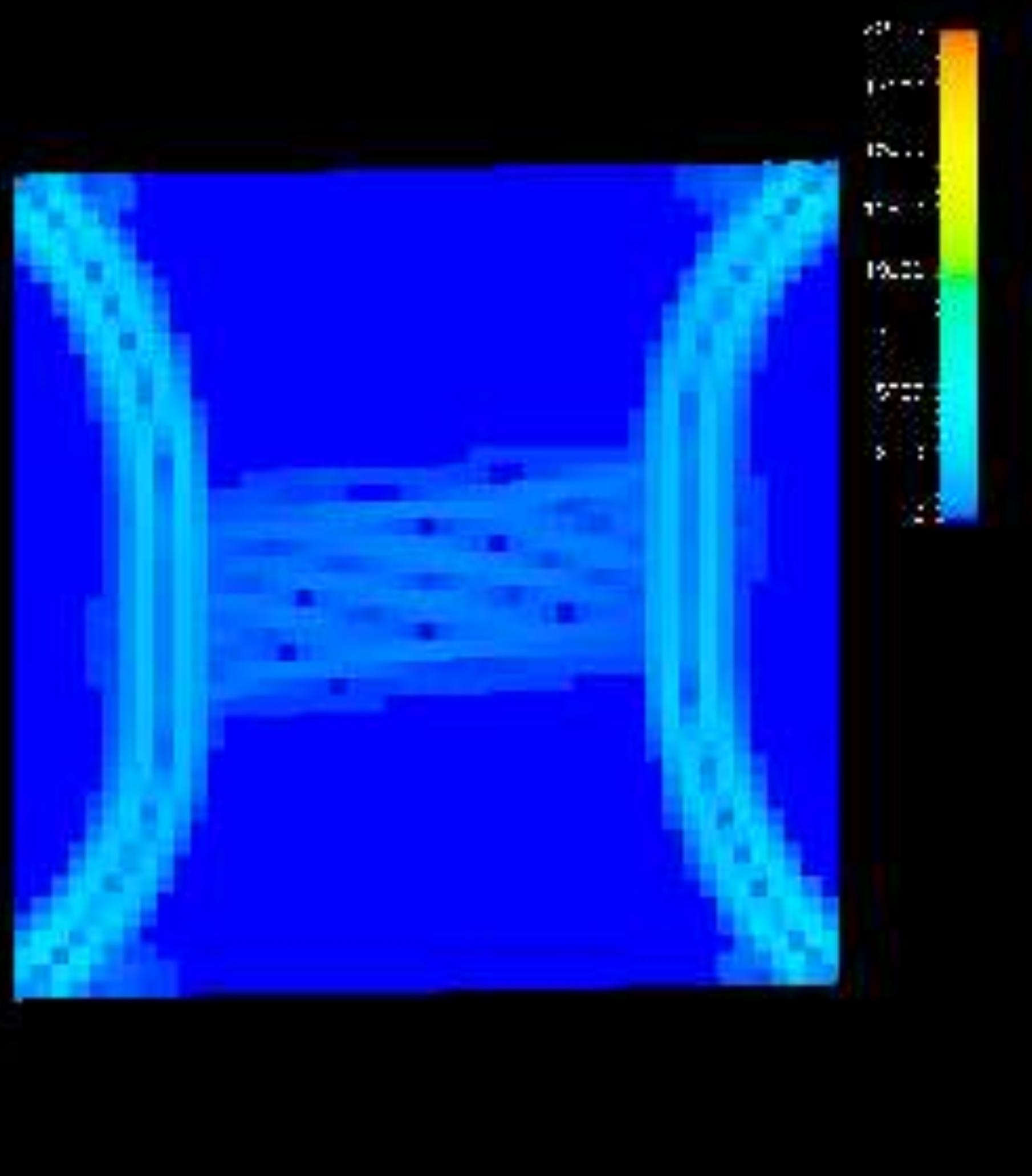$$
\text { . }
$$

Figure $4 \mathrm{e}$

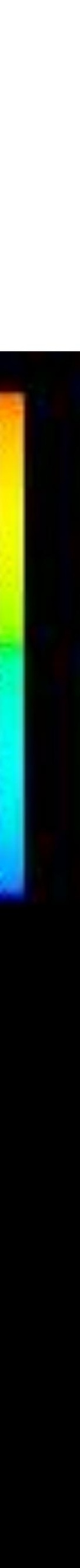


Figure $4 f$

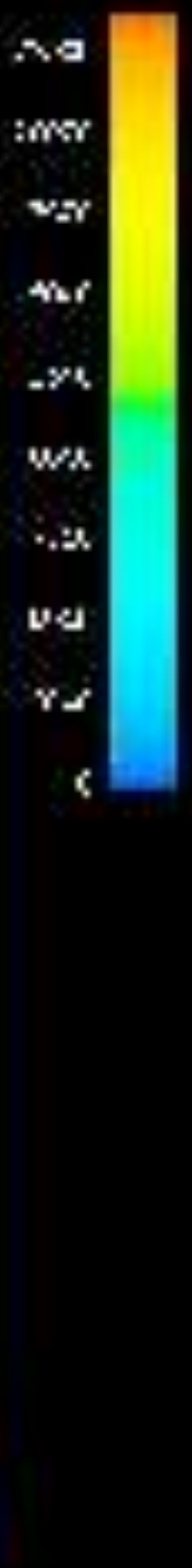




\section{Figure 5a}

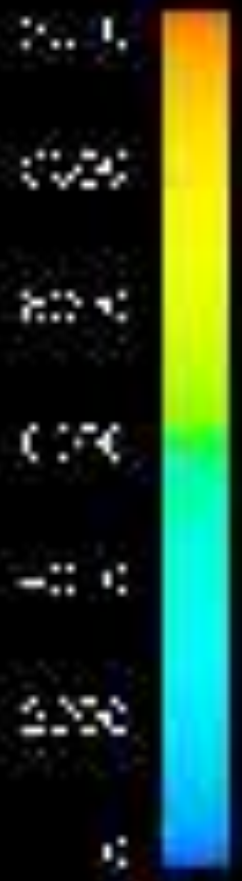

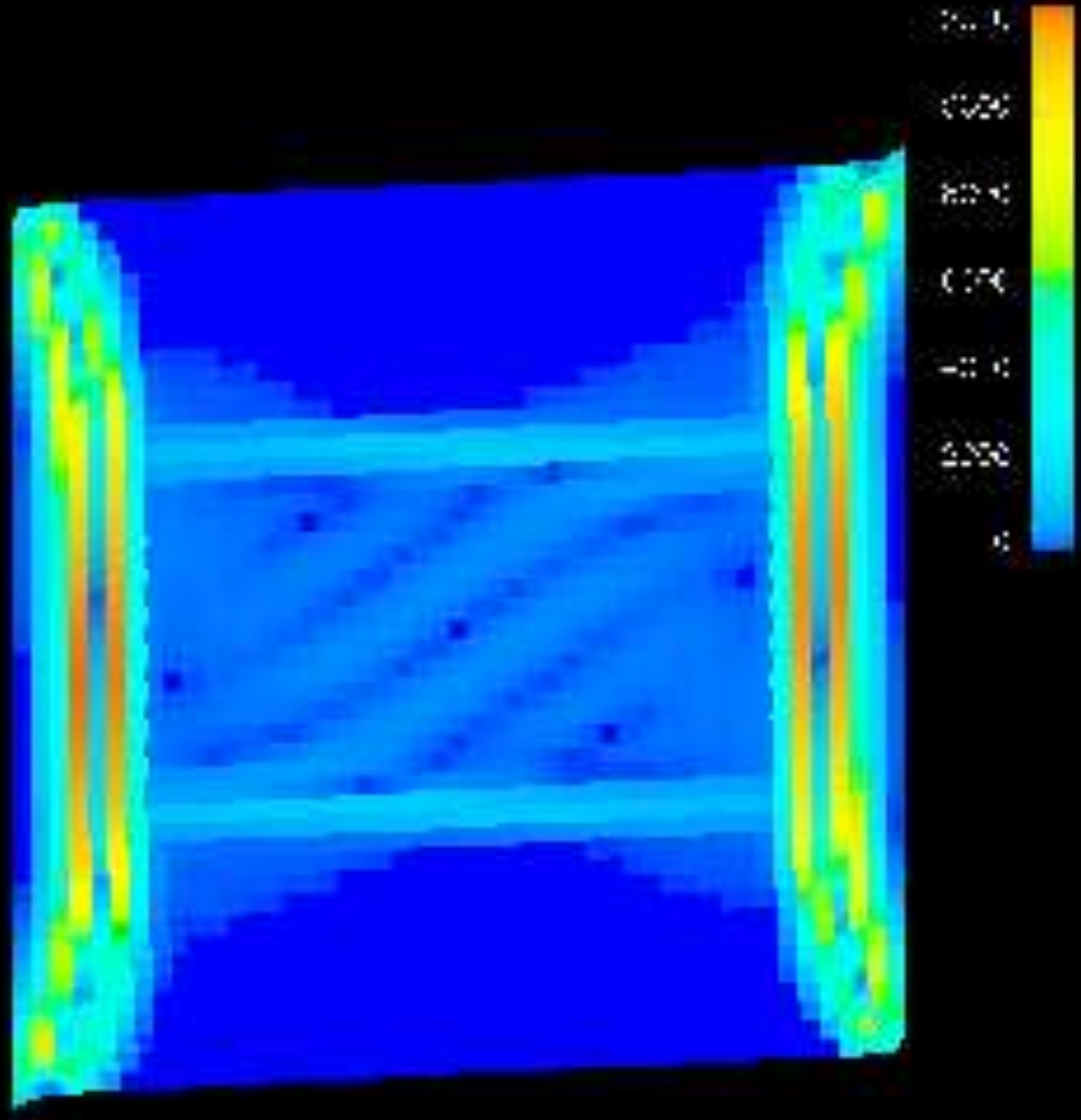


Figure $5 b$
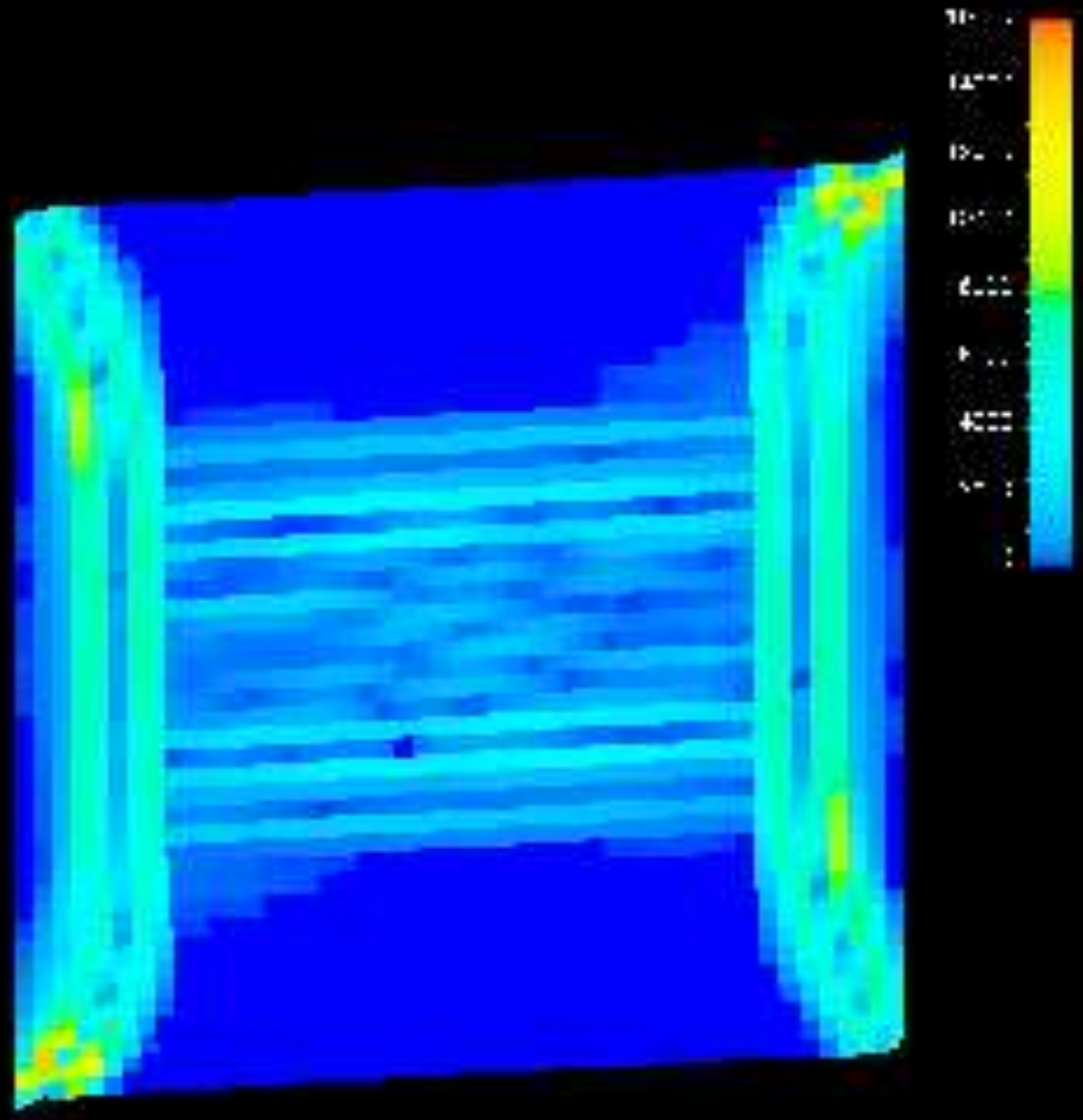
Figure $5 c$

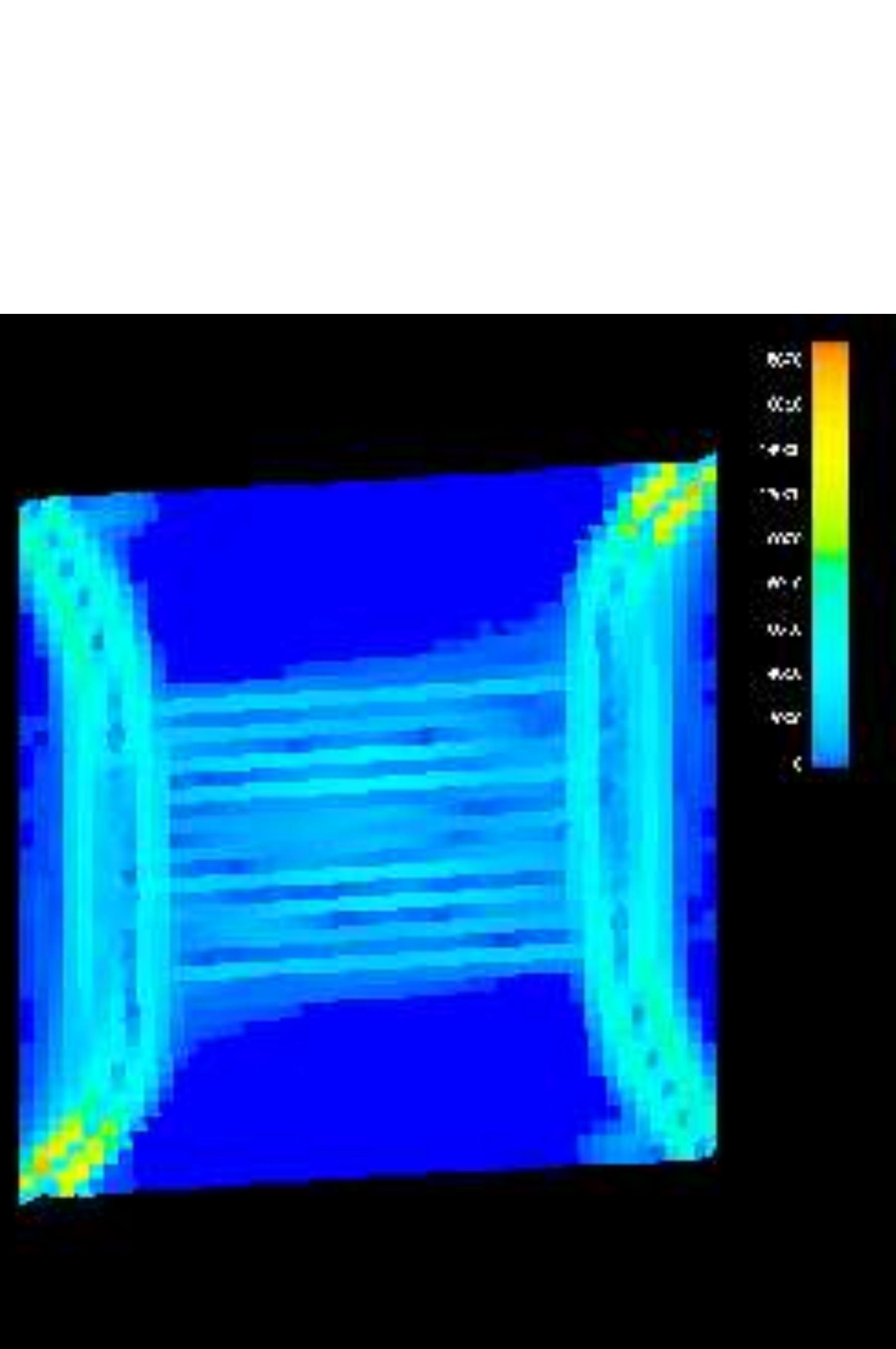

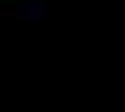

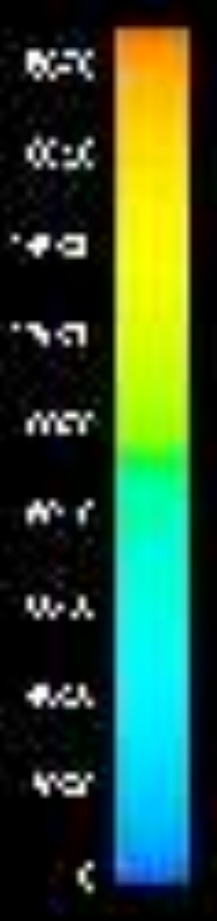

Figure 5 c

c




\section{Figure 5d}
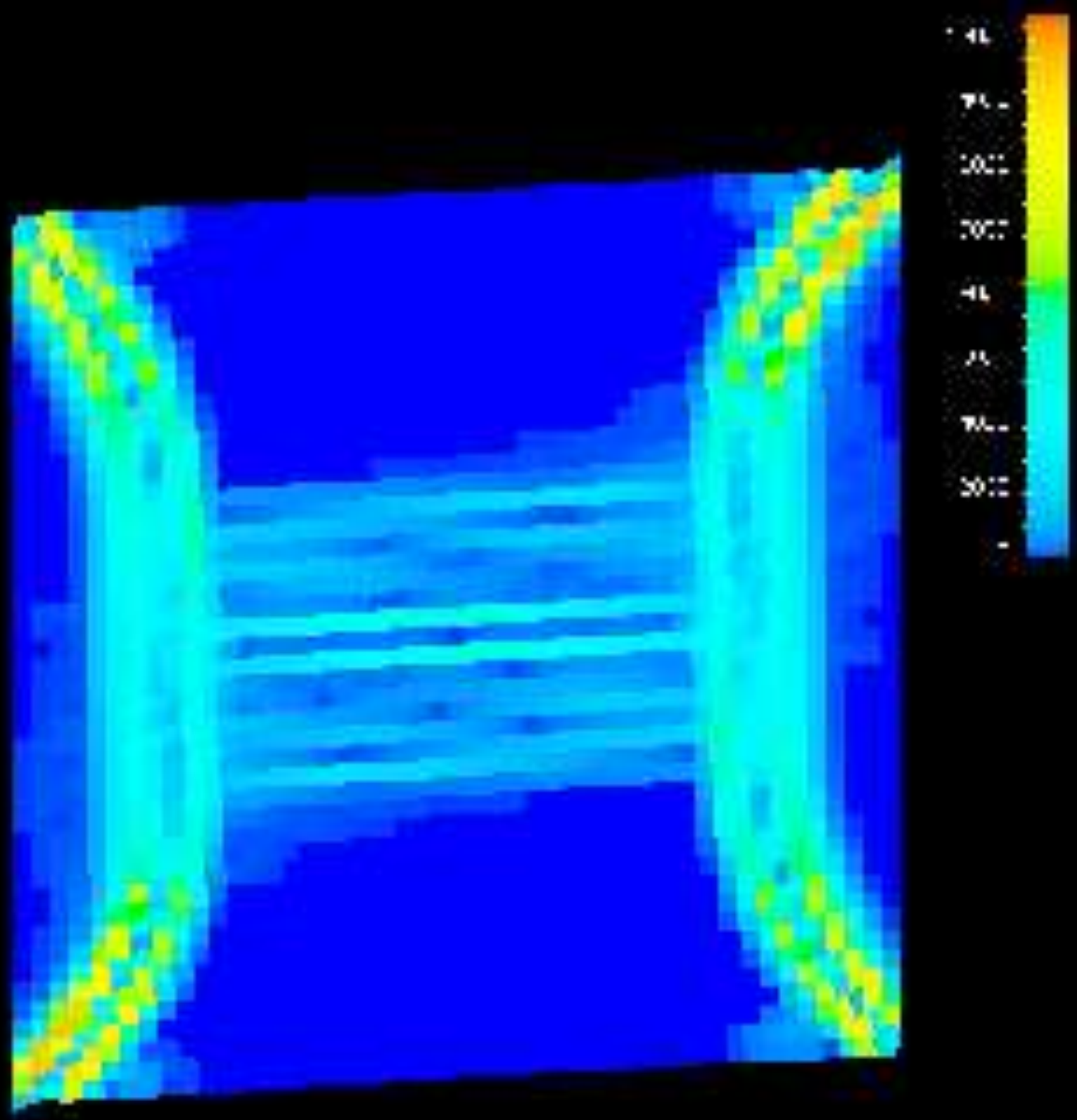

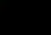

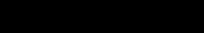


Figure $5 e$
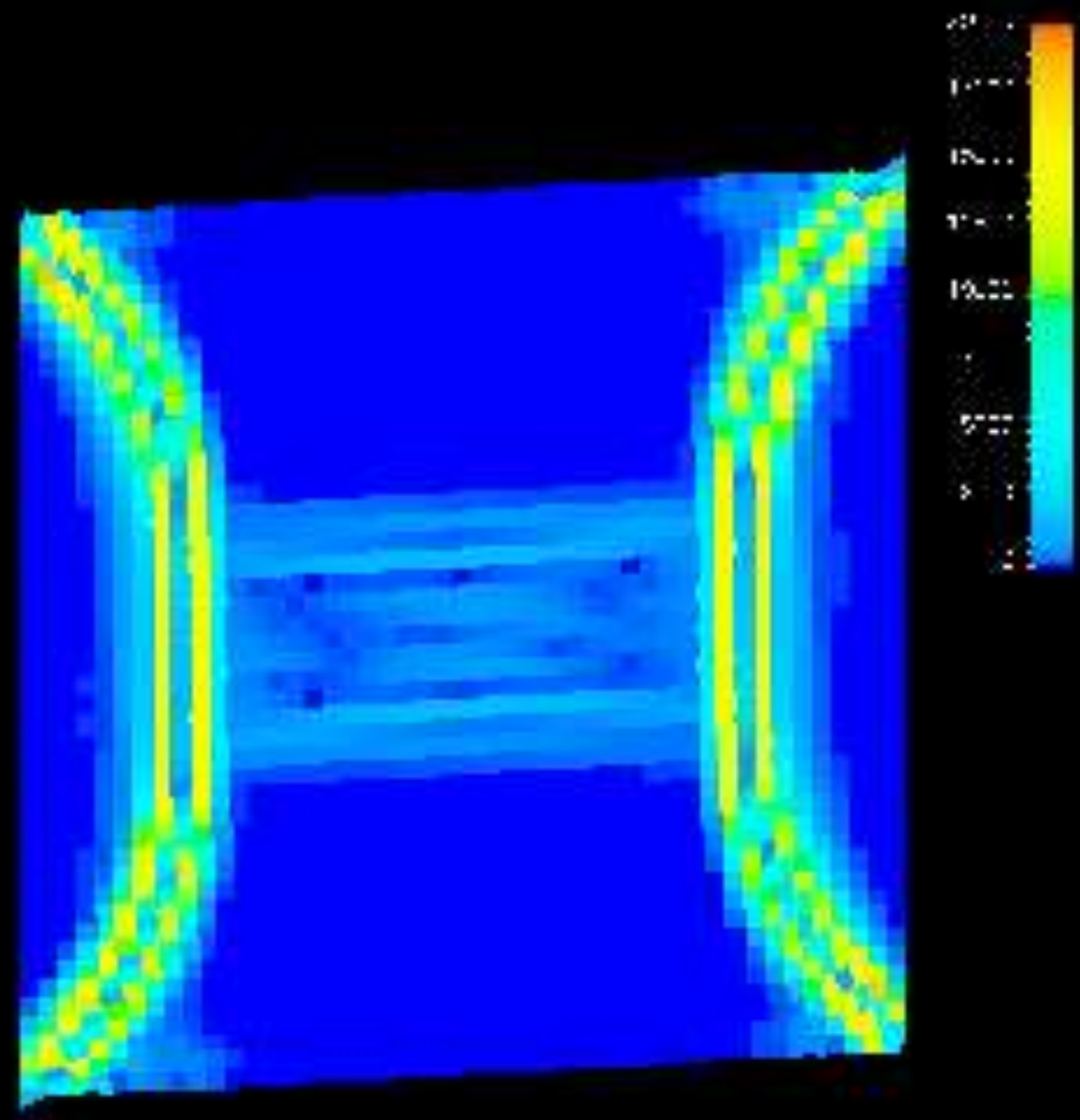


\title{
Figure $5 f$

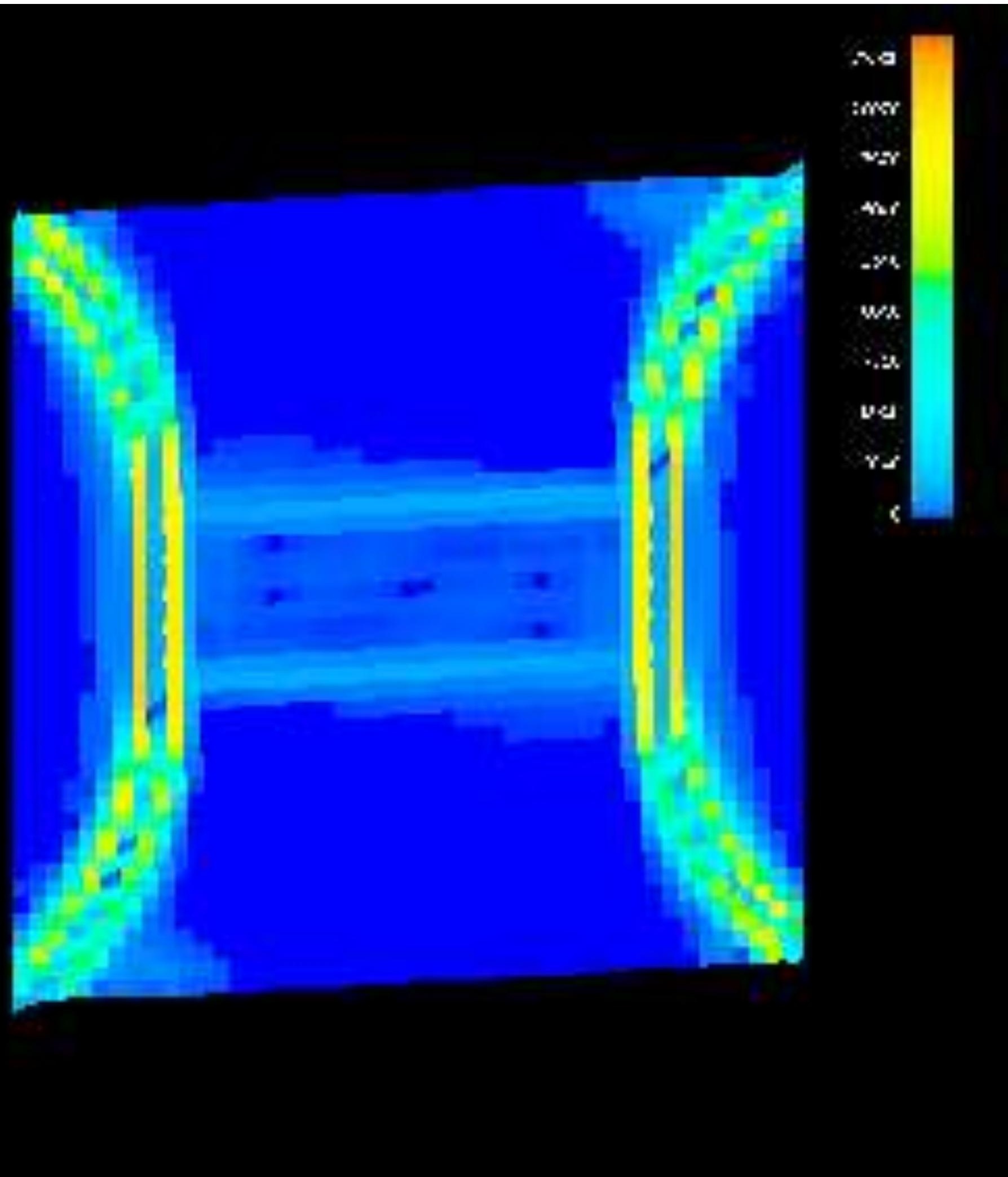

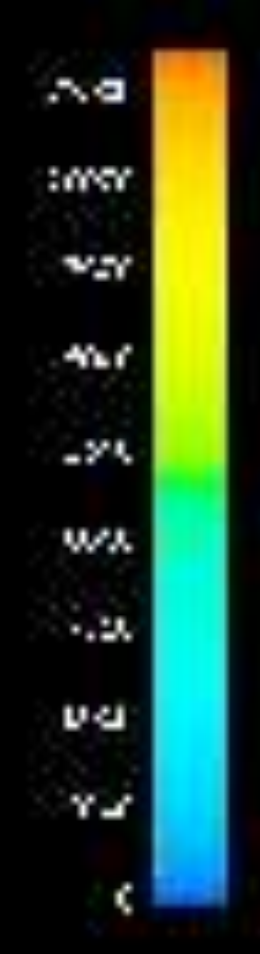

\author{
5
}

